CERN-TH.6904/93/Rev.

\title{
Planckian Energy Scattering and Surface Terms in the Gravitational Action
}

\author{
M. Fabbrichesi \\ CERN, Theory Division \\ CH-1211 Geneva 23, Switzerland \\ R. Pettorino \\ Dipartimento di Scienze Fisiche, Università di Napoli \\ I-80125 Naples, Italy \\ G. Veneziano \\ CERN, Theory Division \\ CH-1211 Geneva 23, Switzerland \\ G.A. Vilkovisky \\ Lebedev Physical Institute and Research Center in Physics \\ Leninsky Prospect 53, Moscow 117924, Russia
}

\begin{abstract}
We propose a new approach to four-dimensional Planckian-energy scattering in which the phase of the $\mathcal{S}$-matrix is written - to leading order in $\hbar$ and to all orders in $R / b=G s / J$ in terms of the surface term of the gravitational action and of a boundary term for the colliding quanta. The proposal is checked at leading order in $R / b$ and also against some already known examples of scattering in strong external gravitational fields.
\end{abstract}

CERN-TH.6904/93/Rev.

November 1993 


\section{Introduction and Outline}

Planckian-energy collisions represent a problem ideally located between the interests of particle physics and those of general relativity. At those energies, large gravitational fields are both generated and felt by the colliding particles, which can then be used as sources and probes for classical and quantum gravity effects.

By changing the impact parameter $b$ of the collision (or its total angular momentum, $J=b E$ ) one can explore large, intermediate or even short distances, thus progressively increasing the sensitivity to quantum effects.

This is particularly noticeable when the problem is studied within the context of string theory. String theory possesses a fundamental length parameter of its own

$$
\lambda_{s} \equiv \sqrt{\alpha^{\prime} \hbar}
$$

While General Relativity expectations are recovered at large distances [1], substantial quantum-string modifications occur [2] at $b<\lambda_{s}$ provided $R \equiv G E<\lambda_{s}$.

On the other hand, for $R>\lambda_{s}$, string-size effects appear to be negligible [1]. In this paper we shall assume to be working in the latter regime, so that one is left with just two dimensionless parameters characterizing the collision:

$$
\alpha_{G} \equiv G s / \hbar \text { and } G s / J=4 R / b .
$$

By definition of Planckian-energy scattering, the parameter $\alpha_{G}$, the gravitational equivalent of the fine-structure constant, will always be taken to be large. This condition ensures that the scattering amplitude has a large phase, hence that the process is semiclassical. We note that this condition also implies that $R$ is much larger than both the Compton wavelength of the colliding particles, $\lambda_{c}=\hbar / E$, and the Planck length, $l_{P} \equiv \sqrt{G \hbar}$.

By contrast, we can still vary at will the second, crucial parameter, $R / b$. While the process is always semiclassical, very different physics is expected to emerge depending on the value of $R / b$.

The simplest case occurs at $R / b \ll 1$. Here a leading eikonal result has been obtained by a variety of methods [1, 3, 4, 5, 6]. We note in particular, for later comparison, the approach of Verlinde and Verlinde [5], where the problem is reduced to estimating a "topological" action, that is, a surface term coming from a reduced two-dimensional action.

Unfortunately, the most interesting semiclassical phenomena that should originate from the collision - such as gravitational collapse, black-hole formation and 
Hawking evaporation - are only expected to occur at values of $R / b$ of $\mathcal{O}(1)$ or less. This can be seen in various ways, either within an $\mathcal{S}$-matrix approach (which gives [7, 6] higher-order corrections as a power series in $R / b$ ) or from the General Relativity point of view (numerical studies of gravitational collapse of rotating systems [8], collisions of black holes at high energy and small impact parameters [9, 10], etc.).

It has been pointed out [11] that the approach of ref. [5] cannot be extended in a straightforward way so as to be able to cope with this interesting regime. So far, the most promising way to tackle this difficult problem is, in our opinion, the one of ref. [7], where, following work by Lipatov [12], one tries to describe the whole series of corrections in $R / b$ in terms of the classical solutions of an effective two-dimensional action (in the transverse coordinates).

The main criticism that one can raise to such an approach is its insistence in separating transverse from longitudinal coordinates even in the regime of large angle scattering, which necessarily has to precede that of collapse. Besides, the approach is technically quite complicated and, so far, only the next-to-leading correction has been computed and found to agree with previous direct calculations.

In this paper we propose a completely new approach, which remains genuinely four-dimensional at all stages and yet yields a "topological" result for the $\mathcal{S}$-matrix. This approach is built upon two main ingredients:

- The correct treatment of the gravitational action through the inclusion of an appropriate surface term;

- A first quantized path integral approach to quantum-field theory developed by Fradkin some thirty years ago.

It makes it possible to express the semiclassical phase, to all orders in $R / b$, just using surface terms. These come partly from the surface term of the gravitational action and partly from a boundary term connected to the external particles.

The outline of the paper is as follows: In section 2 we recall some basic facts about the necessity and form of the surface term to be added to the usual EinsteinHilbert action. We also present some general results on how to express such a surface integral for asymptotically flat space-times in terms of Bondi masses. Details of the calculations pertaining to this section can be found in the Appendix. In section 3 we use Fradkin's approach to quantum field theory in order to give 
convenient expressions for the full and amputated scalar propagators in an external gravitational field. The eikonal approximation is recovered in the relevant limit, but we shall not be restricted to it in the following. In section 4 we discuss scattering in an external gravitational field, easily recovering known results. We deal, in particular, with the case of an external shock-wave and with that of Schwarzschild's metric. In section 5 we combine our previous results to obtain a simple expression for the $\mathcal{S}$-matrix of two colliding particles, first in the case of elastic scattering and then with the inclusion of gravitational bremsstrahlung. In both cases the process is completely determined by surface terms. For the case of elastic scattering we see the external metric approximation emerging at leading order (and failing beyond) and we recover in an elegant way the leading eikonal result. Section 6 contains some remarks concerning the possibility of extending the method to the case in which graviton loops are included.

\section{The gravitational action and the $\mathcal{S}$-matrix of massless particles}

Two related facts distinguish the theory of gravitational interaction in an asymptotically flat space-time. First, the Einstein-Hilbert Lagrangian is linearly homogeneous in the metric, whereas the usual field-theoretic Lagrangians are essentially quadratic in the field. Second, the energy of gravitating fields is given by a surface contribution at the asymptotically flat infinity, whereas, in the nongravitational field theories, it is the integral of a volume density. These two properties have not received proper attention in quantum theory. The reason is probably that, in perturbation theory, the gravitational interaction is really not too different from the others. It seems, however, that any reasonable attempt at non-perturbative quantum gravity should rely on the above basic facts. No such attempt will be undertaken in the present work but we shall argue that, even in the regime where the

gravitational field is treated classically, the use of the above features of gravity can be fruitful. 


\subsection{The gravitational action}

We start by recalling that the Einstein equations with asymptotically flat boundary conditions do not follow from the Einstein-Hilbert action!

$$
S_{E}=-\frac{c^{3}}{16 \pi G} \int \mathrm{d}^{4} x \sqrt{-g} \mathcal{R} .
$$

Indeed, by making a variation of $g_{\mu \nu}$ in a compact domain $\Omega$, one finds

$$
\begin{aligned}
& \delta \int_{\Omega} \mathrm{d}^{4} x \sqrt{-g} \mathcal{R}= \\
& \quad \int_{\Omega} \mathrm{d}^{4} x \sqrt{-g}\left[\left(g^{\mu \nu} \nabla^{2} \delta g_{\mu \nu}-\nabla^{\mu} \nabla^{\nu} \delta g_{\mu \nu}\right)-\left(\mathcal{R}^{\mu \nu}-\frac{1}{2} g^{\mu \nu} \mathcal{R}\right) \delta g_{\mu \nu}\right]
\end{aligned}
$$

where $\nabla^{2}=g^{\alpha \beta} \nabla_{\alpha} \nabla_{\beta}$. The total derivative in (2.2) can be written in terms of contributions from the boundary $\partial \Omega$, but when the spatial boundary is pushed to infinity with the appropriate asymptotic behaviour of the metric, these contributions do not vanish. For the Einstein equations to extremize the action, one should add to the Einstein-Hilbert action some functional of the metric to compensate the contribution of the total derivative in (2.2). We denote this functional by $S_{K}[g]$. The total action is thus of the form

$$
S=-\frac{1}{16 \pi G} \int_{\Omega} \mathrm{d}^{4} x \sqrt{-g} \mathcal{R}+S_{K}[g]+S_{\text {Source }}
$$

where we have included possible matter sources of the gravitational field. The only condition defining $S_{K}[g]$ is that

$$
\delta S_{K}[g]=\frac{1}{16 \pi G} \int_{\Omega} \mathrm{d}^{4} x \sqrt{-g}\left(g^{\mu \nu} \nabla^{2} \delta g_{\mu \nu}-\nabla^{\mu} \nabla^{\nu} \delta g_{\mu \nu}\right)
$$

which ensures that the variational equations of the action (2.3) are of the form

$$
\begin{aligned}
\mathcal{R}^{\mu \nu}-\frac{1}{2} g^{\mu \nu} \mathcal{R} & =-8 \pi G \mathcal{T}^{\mu \nu} \\
\mathcal{T}^{\mu \nu} & =\frac{2}{\sqrt{-g}} \frac{\delta S_{\text {Source }}}{\delta g_{\mu \nu}} .
\end{aligned}
$$

Since the action is defined up to an additive constant, we impose also the normalization condition that $S_{K}[g]$ vanishes when the metric is flat.

The derivatives acting on $\delta g_{\mu \nu}$ in (2.4) can be decomposed into normal and tangential components with respect to the boundary $\partial \Omega$. Terms with tangential

\footnotetext{
${ }^{\dagger}$ We use the signature $(-,+,+,+)$, the conventions $\mathcal{R}_{\nu \mu \alpha}{ }^{\beta}=\partial_{\nu} \Gamma_{\mu \alpha}^{\beta}-\ldots, \mathcal{R}_{\nu \mu}=\mathcal{R}_{\nu \beta \mu}{ }^{\beta}$, $\mathcal{R}=g^{\mu \nu} \mathcal{R}_{\mu \nu}$, and, in what follows, put $c=\hbar=1$.
} 
derivatives can be added and subtracted at will because the action (2.3) is varied under the condition

$$
\left.\delta g_{\mu \nu}\right|_{\partial \Omega}=0
$$

For this reason, there are many functionals $S_{K}[g]$ satisfying the above requirements, differing from each other by tangential derivative terms. Addition of such terms affects neither the variational principle nor the value of the action (2.3) computed with an asymptotically flat metric. The action (2.3) with $\Omega$ extended to the whole of space-time is thus unique.

By using the freedom in tangential derivative terms, the functional $S_{K}[g]$ can be put in an elegant form [13], where it is the trace $K$ of the second fundamental form $K_{i j}$ on $\partial \Omega$ integrated over the boundary. However, we will not be able to use this expression, at least directly, because, as discussed below, we have to deal with null boundaries. We use instead the simplest expression for $S_{K}[g]$, the one that can be obtained as follows.

Let the boundary $\partial \Omega$ be given by the equation

$$
\partial \Omega: \tau(x)=0
$$

where $\tau(x)$ is a piecewise smooth function, and let the sign of $\tau(x)$ be chosen so that the direction of growing $\tau$ leads out of $\Omega$. Then the Gauss theorem can be put in the form:

$$
\int_{\Omega} \mathrm{d}^{4} x \sqrt{-g} \nabla_{\mu} f^{\mu}=\int \mathrm{d}^{4} x \sqrt{-g} \delta(\tau(x)) f^{\mu} \nabla_{\mu} \tau,
$$

which is also valid for null boundaries, $(\nabla \tau(x))^{2}=0$. Accordingly, eq. (2.4) takes the form:

$$
\delta S_{K}[g]=\frac{1}{16 \pi G} \int \mathrm{d}^{4} x \sqrt{-g} \delta(\tau(x)) \nabla_{\mu} \tau\left(g^{\alpha \beta} \nabla^{\mu} \delta g_{\alpha \beta}-g^{\mu \alpha} \nabla^{\beta} \delta g_{\alpha \beta}\right) .
$$

Since the metric enters $S_{K}$ only near the boundary, and the boundary will be carried to the domain where space-time is asymptotically flat, we can introduce an auxiliary flat metric $\tilde{g}_{\mu \nu}$ in this domain and expand $S_{K}$ as

$$
S_{K}=S_{K}[\tilde{g}+h]=A[\tilde{g}]+B[\tilde{g}, h]+\mathcal{O}\left[h^{2}\right]
$$

where $A$ is the zeroth-order term, $B$ the first-order term, and $\mathcal{O}\left[h^{2}\right]$ higher-order terms. With the above normalization,

$$
A[\tilde{g}]=0
$$


and the $\mathcal{O}\left[h^{2}\right]$ terms fall off at infinity too rapidly to give a finite contribution. The result is that, whatever $S_{K}$ is, it is given by a linear term of its deviation from the flat space-time value. But then we already know it, it is:

$$
S_{K}=\frac{1}{16 \pi G} \int_{\substack{\text { asymptotic } \\ \text { domain }}} \mathrm{d}^{4} x \sqrt{-\tilde{g}} \delta(\tau(x)) \nabla_{\mu} \tau\left(\tilde{g}^{\alpha \beta} \tilde{\nabla}^{\mu} h_{\alpha \beta}-\tilde{g}^{\mu \alpha} \tilde{\nabla}^{\beta} h_{\alpha \beta}\right),
$$

where the quantities and operators with tilde refer to the flat metric $\tilde{g}_{\mu \nu}$, and

$$
h_{\mu \nu}=g_{\mu \nu}-\tilde{g}_{\mu \nu} .
$$

The components of $g_{\mu \nu}$ are calculated in a chart covering the asymptotic domain, and the components of $\tilde{g}_{\mu \nu}$ are their flat space-time limits.

The reason for the surface term (2.12) to be non vanishing lies in the fact that the expansion of the Einstein-Hilbert action in the wave field (2.13) starts with a linear term. Hence, the surface term to be subtracted is also linear in the field and, finally, the field itself is not decreasing sufficiently fast at large distance for giving a vanishing contribution.

The boundary contribution to the gravitational action has been discussed in the literature mainly in the context of Euclidean gravity. We need to calculate it for a Lorentzian asymptotically flat space-time. A major requisite of this calculation is the determination of the boundary of an asymptotically flat space-time. If one thinks of space-time as of a "cylinder" bounded by time-like and space-like hypersurfaces, then, at the space-like portions, the metric will not be asymptotically flat at all. The boundary of an asymptotically flat space-time emerges after the Penrose conformal transformation, bringing infinity to a finite distance [14]. It then consists of the spatial infinity $\left(I^{\circ}\right)$, where the space-like geodesics begin and end, the past and future time-like infinities $\left(I^{-}\right.$and $\left.I^{+}\right)$, where the time-like geodesics begin and end, and the past and future null infinities $\left(\mathcal{I}^{-}\right.$and $\left.\mathcal{I}^{+}\right)$, where the null geodesics begin and end. In the physical space-time, the $I^{\circ}, I^{ \pm}, \mathcal{I}^{ \pm}$are understood as infinite limits of affine parameters along the respective geodesics. The metric is asymptotically flat at $\mathcal{I}^{-}, \mathcal{I}^{+}$and $I^{\circ}$, but not at $I^{-}$and $I^{+}$. An important fact is, however, that $I^{\circ}, I^{-}$and $I^{+}$are single points, whereas $\mathcal{I}^{-}$and $\mathcal{I}^{+}$are three-dimensional (null) hypersurfaces. Since the surface term in the action is an integral over the boundary, we conclude that it is the integral over

$$
\partial \Omega=\mathcal{I}^{-} \cup \mathcal{I}^{+}
$$


If space-time has event horizons, the latter also appear as portions of the boundary. We shall come back to this point when discussing the $\mathcal{S}$-matrix.

More knowledge about the boundary is given by the behavior of the metric in its neighborhood. The past and future null infinities are treated similarly. In the appendix we shall describe $\mathcal{I}^{+}$following Sachs [15], and build the metric near $\mathcal{I}^{+}$by considering the congruence $u(x)=$ const, $(\nabla u)^{2} \equiv 0$, of light rays reaching $\mathcal{I}^{+}$. A similar procedure can be followed at $\mathcal{I}^{-}$. In the same appendix, we then calculate the boundary term in the action and show that it can be written in the form

$$
S_{K}=-\frac{1}{2}\left(\int_{-\infty}^{\infty} \mathrm{d} u M_{+}(u)-\int_{-\infty}^{\infty} \mathrm{d} v M_{-}(v)\right),
$$

where $M_{+}(u)$ and $M_{-}(v)$ are the Bondi masses at $\mathcal{I}^{+}$and $\mathcal{I}^{-}$, the precise definition of which is given in the appendix, and the retarded and advanced time are normalized by the conditions (A.18) and (A.32).

\subsection{The $\mathcal{S}$-matrix of massless fields}

The $\mathcal{S}$-matrix of the gravitational field coupled to a set of matter fields $\psi$ is given by the functional integral

$$
\mathcal{S}=\int \mathrm{d}\left[g_{\mu \nu}, \psi\right] e^{i S},
$$

where $S$ is the full action (2.3), and the integration is carried out over all fields interpolating between the asymptotic fields with fixed operator data. For massless conformal invariant fields, these data are at $\mathcal{I}^{ \pm}$. It is assumed that the measure in (2.16) includes all gauge-fixing, ghost and $\delta(0)$ contributions.

To leading order in $\hbar$, the classical path dominates the functional integral, and the $\mathcal{S}$-matrix takes the form

$$
\mathcal{S}_{\text {tree }}=\exp \left(\left.i S\right|_{\text {sol'n }}\right),
$$

where the action is to be calculated on the solution of its variational equations with the appropriate boundary conditions. This approximation amounts to a resummation of the Feynman diagrams illustrated in fig. 1, as discussed further in section 5 .

Consider first the case of pure gravity. By virtue of the field equations (2.5), the volume density of the Lagrangian in (2.3) vanishes, and the action reduces to the surface term only:

$$
\mathcal{S}_{\text {tree }}=\exp \left(\left.i S_{K}[g]\right|_{\text {sol'n }}\right) .
$$


Then, by using the result (2.15), we obtain:

$$
\mathcal{S}_{\text {tree }}=\exp \left[-\frac{i}{2}\left(\int_{-\infty}^{\infty} \mathrm{d} u M_{+}(u)-\int_{-\infty}^{\infty} \mathrm{d} v M_{-}(v)\right)\right]
$$

which relates the $\mathcal{S}$-matrix elements directly to the Bondi masses of the classical gravitational radiation.

The vanishing of the Einstein-Hilbert action in (2.17) is, of course, based on its being homogeneous in the metric. However, in general, homogeneity alone is not sufficient for the action to vanish. With $S(\varphi)$ homogeneous of degree $n$ in $\varphi$, and $\varphi$ infinite-dimensional (e.g. a field), one can only conclude that:

$$
n S(\varphi)=\int \mathrm{d} x \varphi(x) \frac{\delta S(\varphi)}{\delta \varphi}+\text { a surface term } .
$$

The key point here is that the equations of motion are obtained from variations of $\varphi$ which vanish at the boundary, while the variation of $\varphi$ needed in the homogeneity (Euler) equation for $S$, being global, does not satisfy this property. With the corrected action (2.3) for pure gravity, one arrives at the result (2.18), that is to the identification of the surface term in $(2.20)$ with $S_{K}$. We stress once more that the reason for the surface term to be non-vanishing in this case lies in the fact that, for gravity, the surface term is linear in a field which is not decreasing sufficiently fast at large distance.

If one considers, instead of the $\mathcal{S}$-matrix, the generating functional for Green's functions

$$
Z[J]=\int \mathrm{d}\left[g_{\mu \nu}, \psi\right] \exp \left(i S+i \int \mathrm{d}^{4} x \sqrt{-g} g^{\mu \nu} J_{\mu \nu}\right)
$$

and chooses the field variables as $g_{\mu \nu}$ or $\sqrt{-g} g^{\mu \nu}$, the result will again be of the form (2.19), with the only difference that the classical equations will be modified by the presence of an external source. The total volume density of the Lagrangian in (2.21) is again linearly homogeneous in $g_{\mu \nu}$, and no additional surface terms appear. With other choices of the field variables, the result (2.19) will hold only on shell.

The result (2.19) remains valid if the gravitational field is coupled to non-selfinteracting conformal invariant matter fields. Indeed, the quadratic actions of these fields are reduced to surface terms by their own equations of motion, and, unlike the gravitational surface term, they vanish provided one chooses the solutions of the source-free equations that vanish at infinity and have finite energies. Since the energy-momentum tensor of conformal invariant fields is traceless, the total action 
is again of the form (2.18) although the solution to be used in (2.18) is, of course, different. The absence of self interactions combined with conformal invariance of matter fields is a sufficient but not necessary condition for the action to reduce to the surface term $S_{K}[g]$. For example, the action

$$
-\frac{1}{2} \int \mathrm{d}^{4} x \sqrt{-g} g^{\mu \nu} \nabla_{\mu} \psi \nabla_{\nu} \psi
$$

of a scalar field $\psi$ is not conformal invariant but is linearly homogeneous in $g_{\mu \nu}$, so that (2.18) again holds.

A necessary condition follows from the form of expression (2.19) [?. Note that $M_{+}(u)$ has finite limits at both $u=-\infty$ and $u=+\infty$, and similarly $M_{-}(v)$. The limits $M_{+}(-\infty)$ and $M_{-}(+\infty)$ are both equal to the $A D M$ mass $M_{0}$, so that $M_{0}$ cancels in the difference. If matter consists only of massless radiation that comes in through $\mathcal{I}^{-}$and goes out through $\mathcal{I}^{+}$, one has $M_{-}(-\infty)=0$ and $M_{+}(+\infty)=0$. Generally, for the difference of the integrals in (2.19) to be finite, one should at least have

$$
M_{-}(-\infty)=M_{+}(+\infty),
$$

which means that the energy carried by time-like sources is conserved separately. If this condition does not hold, there should be a non-vanishing volume density of the Lagrangian to secure the finiteness of the total action.

A promising feature of writing the action through the Bondi masses, is that it easily allows for the introduction of the machinery and interpretations of classical gravity theory, thus making possible the use of solutions [9, 8, 10] that go far beyond flat-space perturbation theory. The $\mathcal{S}$-matrix can then be constructed and used, hopefully, for studying the semiclassical phenomena discussed in the Introduction. In particular, if the relevant classical solution has event horizons, then not all the energy entering through $\mathcal{I}^{-}$will appear at $\mathcal{I}^{+}$; a portion of it will fall into the black hole. Since the $\mathcal{S}$-matrix is calculated only between the states defined at $\mathcal{I}^{-}$and $\mathcal{I}^{+}$, this loss should be felt in (2.19). Alternatively, one may include the horizon as a portion of the boundary, which means adding the states defined at the horizon.

\footnotetext{
${ }^{\dagger}$ One of the authors (G.A.V.) is grateful to Don Page for discussing this point.
} 


\section{Scalar Propagators in Fradkin's Approach}

Fradkin [16] has pioneered an approach to quantum field theory, in which integration over a quantum field is avoided by writing the propagators as functional integrals of the first quantized theory. Here we use his formulation in the case of the propagator for a scalar field in Einstein's theory of gravity and in the presence of an electromagnetic field.

\subsection{The Propagator}

The Green function (Feynman propagator) for a scalar field is defined [17] as

$$
G(x, y / g)=\left\langle y\left|\mathcal{H}^{-1}\right| x\right\rangle=i \int_{0}^{\infty} \mathrm{d} \nu\langle y|\exp [-i(\mathcal{H}-i \epsilon) \nu]| x\rangle,
$$

where, in the case of propagation in an external gravitational field $g_{\mu \nu}$,

$$
2 \mathcal{H}=-\nabla_{\mu} g^{\mu \nu} \nabla_{\nu}+m^{2}=\frac{-1}{\sqrt{-g}} \partial_{\mu} \sqrt{-g} g^{\mu \nu} \partial_{\nu}+m^{2} .
$$

Equation (3.1) admits a representation as a path integral as 18

$$
G(x, y / g)=i \int_{0}^{\infty} \mathrm{d} \nu \int_{X(0)=x}^{X(\nu)=y}\left[\mathrm{~d} X^{\mu}\right]\left[\mathrm{d} P_{\mu}\right] \exp \left[i \int_{0}^{\nu} \mathrm{d} \nu(P \cdot \dot{X}-\mathcal{H})\right],
$$

where the dependence of $\mathcal{H}$ on $P^{\mu}$ is given by replacing the covariant derivative $\nabla^{\mu} \rightarrow i p^{\mu}$. The correctness of this procedure can also be verified by considering the action of a relativistic point in an external metric:

$$
S=-m \int \mathrm{d} \lambda \sqrt{-g_{\mu \nu} \dot{X}^{\mu} \dot{X}^{\nu}} .
$$

This can also be written as

$$
S=-\frac{1}{2} \int \mathrm{d} \lambda\left[e^{-1 / 2}\left(-g_{\mu \nu} \dot{X}^{\mu} \dot{X}^{\nu}\right)+e^{1 / 2} m^{2}\right]
$$

by introducing the auxiliary einbein $e$. The action (3.5) has the advantage of allowing a straightforward massless limit $m \rightarrow 0$. Eliminating $e$ through its equation of motion:

$$
e=-g_{\mu \nu} \dot{X}^{\mu} \dot{X}^{\nu} / m^{2}
$$

one recovers (3.4). 
According to (3.5), the conjugate momentum is

$$
P_{\mu}=e^{-1 / 2} \dot{X}^{\nu} g_{\mu \nu}
$$

so that, upon use of (3.7) and (3.6):

$$
P_{\mu} g^{\mu \nu} P_{\nu}=g_{\mu \nu} \dot{X}^{\mu} \dot{X}^{\nu} / e=-m^{2} .
$$

The reparametrization invariance of the action (3.4) forces the canonical Hamiltonian to vanish so that the full Hamiltonian reduces to the constraint itself, that is

$$
\mathcal{H}=\frac{1}{2}\left(P_{\mu} g^{\mu \nu} P_{\nu}+m^{2}\right) .
$$

In the definition (3.9) we have neglected ordering ambiguities, which give rise to a possible additional term proportional to the scalar curvature. The coefficient of such a term can be fixed, for example, by imposing conformal invariance for the massless, spin-0 wave equation, to obtain

$$
2 \mathcal{H}=-\nabla_{\mu} g^{\mu \nu} \nabla_{\nu}+m^{2}-\frac{1}{6} \mathcal{R} .
$$

However, in this paper, the functional integral (3.3) will be used only to the lowestorder WKB approximation, where it is unambiguous, and the problems inherent in its definition will not be discussed further.

The propagator is therefore defined by (3.3), which yields, after integrating out the momenta,

$$
\begin{aligned}
& G(x, y / g)=\int_{0}^{\infty} \mathrm{d} \nu \int\left[\sqrt{-g(X)} \mathrm{d} X^{\mu}\right] \delta^{(4)}(x-X(0)) \delta^{(4)}(y-X(\nu)) \\
& \quad \times \exp \left[(i / 2) \int_{0}^{\nu} \mathrm{d} \xi\left(g_{\mu \nu} \dot{X}^{\mu} \dot{X}^{\nu}-m^{2}\right)\right] .
\end{aligned}
$$

The measure in (3.11) and below must be understood as containing the usual factor $(2 \pi \mathrm{d} \nu)^{-2}$ arising in the integration over $\left[\mathrm{d} P_{\mu}\right]$.

The Green function (3.11) can also be generalized in a straightforward fashion to include an electromagnetic field by the usual substitution $p_{\mu} \rightarrow p_{\mu}-e A_{\mu}$ to give

$$
\begin{aligned}
& G(x, y / g, A)=\int_{0}^{\infty} \mathrm{d} \nu \int\left[\sqrt{-g(X)} \mathrm{d} X^{\mu}\right] \delta^{(4)}(x-X(0)) \delta^{(4)}(y-X(\nu)) \\
& \quad \times \exp \left[(i / 2) \int_{0}^{\nu} \mathrm{d} \tau\left(g_{\mu \nu} \dot{X}^{\mu} \dot{X}^{\nu}+2 e A_{\mu} \dot{X}^{\mu}-m^{2}\right)\right] .
\end{aligned}
$$

The propagators (3.11) and (3.12) are connected (in the sense that the vacuum diagrams have been already divided out), but not amputated. In order to properly 
define the $\mathcal{S}$-matrix à la LSZ, we need the on-shell amputated propagator, in which the external legs have been removed:

$$
\begin{aligned}
& G_{c}\left(p, p^{\prime} / g, A\right)= \\
& \lim _{p^{2}, p^{\prime 2} \rightarrow-m^{2}}\left(p^{2}+m^{2}\right)\left(p^{2}+m^{2}\right) \int \mathrm{d}^{4} x \mathrm{~d}^{4} y \exp \left[i p \cdot x-i p^{\prime} \cdot y\right] G(x, y / g, A),
\end{aligned}
$$

where the Fourier transform is taken with respect to asymptotically Cartesian coordinates. The prescription we use for obtaining $G_{c}$ directly is the following [. The integral over $\nu$ in (3.11) and (3.12) is replaced by a limit in which the initial and final $\nu$ 's go, respectively, to minus and plus infinity. Accordingly, the amputated propagator is given by

$$
G_{c}\left(p, p^{\prime} / g, A\right)=\lim _{p^{2}, p^{\prime 2} \rightarrow-m^{2}} \lim _{\substack{\nu_{i} \rightarrow-\infty \\ \nu_{f} \rightarrow \infty}} \frac{1}{\nu_{f}-\nu_{i}} \int\left[\sqrt{-g(X)} \mathrm{d} X^{\mu}\right] \exp [i \mathcal{A}],
$$

where

$$
\mathcal{A}=p \cdot X\left(\nu_{i}\right)-p^{\prime} \cdot X\left(\nu_{f}\right)+\int_{\nu_{i}}^{\nu_{f}} \mathrm{~d} \tau L(\tau)
$$

and

$$
L(\tau)=\frac{1}{2}\left(g_{\mu \nu} \dot{X}^{\mu} \dot{X}^{\nu}+2 e A_{\mu} \dot{X}^{\mu}-m^{2}\right) .
$$

The prescription (3.14) gives the same result as the definition (3.13), as can be readily checked in perturbation theory.

The form (3.16) of the Lagrangian corresponds to the gauge in which $e=1$ and $\lambda=\tau / m$, where $\tau$ is the particle's proper time. Only affine transformations remain as invariances of the action after such a gauge fixing.

The propagator (3.14) is our starting point. It provides a solution for the quantum motion of a scalar particle in terms of a functional integral over trajectories. Notice that the measure $\left[\mathrm{d} X^{\mu}\right]$ in (3.14) includes an integration over the initial $\left(X^{\mu}\left(\nu_{i}\right)\right)$ and final $\left(X^{\mu}\left(\nu_{f}\right)\right)$ positions.

At Planckian energies the mass term as well as the electromagnetic interactions can be neglected. Furthermore the action $\mathcal{A}$ is large and the stationary phase approximation should be valid to $\mathcal{O}\left(\hbar^{-1}\right)$.

\footnotetext{
$\dagger$ This method, based on a result given in [19], was used within a functional approach in a series of papers on scattering amplitudes in QED [20].
} 
Making $\mathcal{A}$ stationary not only with respect to $X^{\mu}(\tau)$ inside the integration region for $\tau$ but also, say, with respect to the final position gives:

$$
\begin{aligned}
& \ddot{X}^{\mu}+\Gamma_{\nu \sigma}^{\mu}(X) \dot{X}^{\nu} \dot{X}^{\sigma}=0, \\
& \dot{X}^{\nu} g_{\mu \nu}\left(\nu_{f}\right)=\dot{X}^{\nu}\left(\nu_{f}\right) \eta_{\mu \nu}=p_{\mu}^{\prime} .
\end{aligned}
$$

At this point the only integration left is an ordinary integration over the initial position, everything else being then fixed by the (null) geodesic. As a result the bulk term in (3.15) vanishes and the phase of the Green function (3.14) (phase shift) can be computed in terms of the initial and final positions of the particle as:

$$
\begin{aligned}
G_{c}\left(p, p^{\prime} / g\right) & \simeq \operatorname{Lim} \int \mathrm{d}^{4} X_{i} \exp i\left[p \cdot X_{i}-p^{\prime} \cdot X_{f}\left(X_{i}\right)\right] \\
& \simeq \operatorname{Lim} \int \mathrm{d}^{4} \Sigma \exp -i[q \cdot \Sigma+P \cdot \Delta(\Sigma)],
\end{aligned}
$$

where we have introduced the notations:

$$
\begin{aligned}
X_{i} \equiv X\left(\nu_{i}\right), & X_{f} \equiv X\left(\nu_{f}\right), \\
\Sigma=\left(X_{i}+X_{f}\right) / 2, & \Delta=X_{f}-X_{i}, \\
P=\left(p+p^{\prime}\right) / 2, & q=p^{\prime}-p,
\end{aligned}
$$

and we have taken $\sqrt{-g\left(X_{i}\right)}=1$ since the asymptotic conditions are defined far enough from any gravitation field and we are using Cartesian coordinates there. Finally, we have introduced the short-hand notation:

$$
\operatorname{Lim} \equiv \lim _{p^{2}, p^{\prime 2} \rightarrow-m^{2}} \lim _{\substack{\nu_{i} \rightarrow-\infty \\ \nu_{f} \rightarrow \infty}} \frac{1}{\nu_{f}-\nu_{i}} .
$$

The final expression for the propagator (3.19) only depends on $X_{i}$ and $X_{f}$ : it has thus been reduced to a "boundary" term. The propagator (3.19) appears to take different forms according to which variables are integrated first by means of the saddle-point approximation (i.e. by using the geodesic) and which are left to the end for a more precise integration method. In (3.19) we have written two possible forms for the propagator that we will use in the following. In the second one, we have traded the remaining integration over $X_{i}$ for the one over $\Sigma$, with the understanding that $\Delta$ should be expressed in terms of $\Sigma$ and of the external field.

The final outcome of such a procedure may depend, in general, on the above separation of integration variables, the correct choice being dictated by the particular 
problem at hand. In the following, in order to be on the safe side, we shall always imply that our final results, when expressed as integrals, are to be trusted only in the saddle-point approximation, where the order of integration becomes immaterial and the result is unambiguous.

\subsection{Eikonal Approximation}

As a check of (3.14) and of (3.19), we can compute $G_{c}$ in the eikonal limit. This corresponds to a saddle-point approximation for the functional integral in which the classical trajectory $X^{\mu}(\tau)$ is computed to the lowest non-trivial order. In this case, the Green function (3.14) feels the change in phase along such a trajectory as a function of the external field $g_{\mu \nu}(X)$ or $A_{\mu}(X)$.

Let us consider again the massless case $m=0$ in the absence of electromagnetism. We write the metric tensor as

$$
g_{\mu \nu}=\eta_{\mu \nu}+2 \kappa h_{\mu \nu}
$$

where $\kappa^{2}=8 \pi G$.

Solving for the geodesic motion (3.17) to lowest order in $\kappa$ yields:

$$
\dot{X}^{\mu}(\nu)=\dot{X}^{\mu}\left(\nu_{i}\right)-\dot{X}^{\nu}\left(\nu_{i}\right) \dot{X}^{\sigma}\left(\nu_{i}\right) \int_{\nu_{i}}^{\nu} \mathrm{d} \tau \Gamma_{\nu \sigma}^{\mu}(X(\tau))
$$

and thus, after use of (3.18),

$$
X^{\mu}(\nu)=X_{i}^{\mu}+p^{\mu}\left(\nu-\nu_{i}\right)-p^{\nu} p^{\sigma} \int_{\nu_{i}}^{\nu} \mathrm{d} \tau \int_{\nu_{i}}^{\tau} \mathrm{d} \tau^{\prime} \Gamma_{\nu \sigma}^{\mu}\left(X\left(\tau^{\prime}\right)\right) .
$$

We can now insert the above perturbative results into (3.15) to obtain

$$
\mathcal{A}=-q \cdot \Sigma+\kappa \int_{\nu_{i}}^{\nu_{f}} h_{\mu \nu}(X(\tau)) p^{\mu} p^{\nu} \mathrm{d} \tau+\mathcal{O}\left(q^{2}\right)+\mathcal{O}(\kappa q) .
$$

In the argument of $h_{\mu \nu}$ we may now use the lowest-order (straight) trajectory:

$$
X_{0}^{\mu}(\nu)=\Sigma^{\mu}+p^{\mu}\left[\nu-\left(\nu_{f}+\nu_{i}\right) / 2\right],
$$

where

$$
p^{\mu}=\frac{\Delta^{\mu}}{\nu_{f}-\nu_{i}} .
$$


Inserting these results into (3.19), the eikonal propagator becomes

$$
\begin{aligned}
& G_{c}\left(p, p^{\prime} / h\right)=\lim _{\substack{\nu_{i} \rightarrow-\infty \\
\nu_{f} \rightarrow \infty}} \frac{1}{\nu_{f}-\nu_{i}} \int \mathrm{d}^{4} \Sigma \\
& \quad \times \exp \left[-i q \cdot \Sigma+i \kappa \int_{\nu_{i}}^{\nu_{f}} h_{\mu \nu}\left(\Sigma+p\left(\tau-\frac{\nu_{f}+\nu_{i}}{2}\right)\right) p^{\mu} p^{\nu} \mathrm{d} \tau\right],
\end{aligned}
$$

The component of $\Sigma$ parallel to $p$ can be easily integrated since the integrand depends trivially on it:

$$
\int \mathrm{d}^{4} \Sigma \exp [-i q \cdot \Sigma](\ldots)=p^{0} \int_{\nu_{i}}^{\nu_{f}} \mathrm{~d} \nu \exp \left[-i \frac{q \cdot p}{2} \nu\right] \int \mathrm{d}^{3} \Sigma(\ldots)
$$

The integration in $\mathrm{d} \nu$ cancels, to leading order in $q$, the factor $1 /\left(\nu_{f}-\nu_{i}\right)$ in the limit defining (3.14) and we are left with the integration over the three components of $\Sigma$ orthogonal to $p$.

After a trivial change of variables we finally obtain:

$$
G_{c}\left(p, p^{\prime} / h\right) \simeq E \int \mathrm{d}^{3} b \exp \left[-i q \cdot b+i \kappa \int_{-\infty}^{+\infty} h_{\mu \nu}(b+p \tau) p^{\mu} p^{\nu} \mathrm{d} \tau\right]
$$

where we have introduced

$$
b=\frac{\nu_{f} X_{i}-\nu_{i} X_{f}}{\nu_{f}-\nu_{i}},
$$

so that $X_{0}^{\mu}(\nu)=b^{\mu}+p^{\mu} \nu$, and the remaining integration is with respect to the components of $b$ others than the one in the direction of $p$. The above result is in agreement with the known expression [21].

The electromagnetic case can also be derived along similar lines to give the old result [22]:

$$
G_{c}\left(p, p^{\prime} / A\right) \simeq E \int \mathrm{d}^{3} b \exp \left[-i q \cdot b+i e \int_{-\infty}^{+\infty} A_{\mu}(b+p \tau) p^{\mu} \mathrm{d} \tau\right] .
$$

It is perhaps instructive to give a second derivation of the gravitational eikonal starting directly from (3.14) and using at first only (3.17) at fixed $X\left(\nu_{i}\right), X\left(\nu_{f}\right)$. A straightforward calculation then gives:

$$
\begin{aligned}
\mathcal{A}= & -P \cdot \Delta-q \cdot \Sigma+\frac{\Delta^{\mu} \Delta^{\nu} \eta_{\mu \nu}}{2\left(\nu_{f}-\nu_{i}\right)} \\
& +\frac{\Delta^{\mu} \Delta^{\nu}}{\left(\nu_{f}-\nu_{i}\right)^{2}} \kappa \int_{\nu_{i}}^{\nu_{f}} \mathrm{~d} \tau h_{\mu \nu}\left(\Sigma+\Delta \frac{\tau-\left(\nu_{f}+\nu_{i}\right) / 2}{\nu_{f}-\nu_{i}}\right) .
\end{aligned}
$$


The integration over $\Delta$ can be done to lowest order in $\kappa$ by standard perturbative techniques. $\Delta$ is replaced in the interaction part of the action by a differential operator acting on the remaining integral, which is Gaussian, to obtain:

$$
\begin{aligned}
& G_{c}\left(p, p^{\prime} / g\right)=\operatorname{Lim} \int \mathrm{d}^{4} \Sigma \exp (-i q \cdot \Sigma) \\
& \quad \times \exp \left[\frac{-i}{\left(\nu_{f}-\nu_{i}\right)^{2}} \frac{\partial}{\partial P_{\mu}} \frac{\partial}{\partial P_{\nu}} \kappa \int_{\nu_{i}}^{\nu_{f}} \mathrm{~d} \tau h_{\mu \nu}(\Sigma+\ldots)\right] \exp \left[-i P^{2}\left(\nu_{f}-\nu_{i}\right) / 2\right] .
\end{aligned}
$$

Terms in (3.34) obtained by not acting on the exponent can be shown to be subleading by powers of $q / P$ or of $1 / P^{2}\left(\nu_{f}-\nu_{i}\right)$ and are thus negligible for small angles. One is therefore left with the differential operators acting only on the exponent and one remaining ordinary integration variable, which can be chosen to be $\Sigma$. The result (3.30) is thus easily recovered.

\section{Scattering in an External Gravitational Field}

\subsection{General Considerations}

Before moving on to the full scattering matrix of the two-body collision, it is useful to check our framework in the simpler case of the scattering in an external gravitational field.

We consider two examples for which an exact solution for the geodesics is known: the (generalized) Aichelburg-Sexl (AS) shock wave [23] and the Schwarzschild (black hole) metric [24]. Planckian scattering in the Schwarzschild metric has been discussed in perturbation theory in [25].

\subsection{Scattering by a null shock wave}

The AS metric is an exact solution for the gravitational field produced by a single massless particle. Its generalization to an instantaneous light pulse of arbitrary energy profile $\rho(y, z)$ is of the form:

$$
\mathrm{d} s^{2}=-\mathrm{d} U \mathrm{~d} V+f(y, z) \delta(U) \mathrm{d} U^{2}+\mathrm{d} y^{2}+\mathrm{d} z^{2},
$$

where $U=t-x, V=t+x$ are flat-space null coordinates and the function $f$ of the transverse coordinates $y, z$, the profile function of the shock wave, is related to the energy profile $\rho$ by the Einstein equations:

$$
\Delta f=-2 \kappa^{2} \rho .
$$


For the special case of a point particle of energy $E_{b}$ ( $b$ for beam), this gives the well known AS result, for which

$$
\rho=E_{b} \delta(U) \delta^{(2)}\left(x_{T}\right)
$$

where $x_{T}=(y, z)$, and

$$
f\left(x_{T}\right)=-\frac{\kappa^{2}}{2 \pi} E_{b} \ln \left(y^{2}+z^{2}\right) .
$$

The classical trajectory lies in a plane. In a coordinate system in which this is the $x-y$ plane it is given, in parametric form, by [26]

$$
\begin{aligned}
U(\nu) & =2 p^{u} \nu+U_{0} \\
V(\nu) & =f^{\prime 2} U(\nu) \theta(U) / 4+f(b) \theta(U)+V_{0} \\
x_{T}(\nu) & =\left(b+f^{\prime} U(\nu) \theta(U) / 2,0\right),
\end{aligned}
$$

where $V\left(\nu_{i}\right)=V_{0}, U\left(\nu_{i}\right)=U_{0}$ and $x_{T}\left(\nu_{i}\right)=b$ are the initial conditions.

We take $\nu_{i}=0$ and $\nu_{f}=\nu$ to simplify the notation; the propagator can be written according to (3.19) as

$$
\begin{aligned}
& G_{c}\left(p, p^{\prime} / A S\right)= \\
& \quad \lim _{\nu \rightarrow \infty} \frac{1}{\nu} \int \mathrm{d}^{4} \Sigma \exp i\left[\left(q^{u} \Sigma^{v}+q^{v} \Sigma^{u}\right) / 2-q_{T} \Sigma_{T}\right. \\
& \left.\quad+\left(P^{u} \Delta^{v}+P^{v} \Delta^{u}\right) / 2-P_{T} \Delta_{T}\right]
\end{aligned}
$$

where, from (4.5), we have:

$$
\begin{aligned}
\Sigma^{v} & =\frac{1}{2}\left[f(b)+\frac{1}{4} f^{\prime 2}\left(2 p^{u} \nu+U_{0}\right)\right]+V_{0}, \\
\Sigma^{u} & =p^{u} \nu+U_{0}, \\
\Sigma_{T} & =\left(b+\frac{1}{4} f^{\prime}\left[2 p^{u} \nu+U_{0}\right], 0\right)
\end{aligned}
$$

and we parametrize the momenta as follows:

$$
\begin{aligned}
P & =\frac{1}{2}\left(E+E^{\prime},-E-E^{\prime} \cos \vartheta,-E^{\prime} \sin \vartheta, 0\right), \\
q & =\left(E^{\prime}-E, E-E^{\prime} \cos \vartheta,-E^{\prime} \sin \vartheta, 0\right) .
\end{aligned}
$$

Integration over $V_{0}$ gives a $\delta\left(q^{u}\right)$ thus enforcing

$$
E=E^{\prime}(1+\cos \vartheta) / 2,
$$


so that

$$
P^{u}=2 E, \quad q^{v}=2 P^{v}=2\left(E^{\prime}-E\right) \quad \text { and } \quad 2 P_{T}=q_{T}=-E^{\prime} \sin \vartheta .
$$

Accordingly, the phase becomes

$$
-q_{T} \cdot b+E f+\left[\left(E^{\prime}-E\right)+\frac{1}{4} f^{\prime 2} E-\frac{1}{2} q_{T} f^{\prime}\right] \widetilde{U}
$$

where $\widetilde{U}=2 p^{u} \nu+U_{0}$.

The result (4.11) can also be written as

$$
-q_{T} \cdot b+E f+\frac{\widetilde{U}}{4 E}\left[q_{T}-f^{\prime} E\right]^{2}
$$

by means of the mass-shell condition

$$
q_{T}^{2}=4 E\left(E^{\prime}-E\right)
$$

The above calculation can be repeated, of course, for any initial transverse vector $x_{T}\left(\nu_{i}\right)=\left(b_{1}, b_{2}\right)$. The Green function is therefore

$$
\begin{aligned}
& G_{c}\left(p, p^{\prime} / A S\right)=\operatorname{Lim} \frac{2 p^{u} \delta\left(q_{u}\right)}{\nu} \int_{0}^{\nu} \mathrm{d} \nu^{\prime} \int \mathrm{d}^{2} b \exp \left[-i q_{T} \cdot b+i E f(b)\right] \\
& \quad \times \exp \left[i \nu^{\prime}\left(q_{T}-f^{\prime} E\right)^{2}\right]
\end{aligned}
$$

which is dominated by a stationary phase at

$$
q_{T}=E f^{\prime}
$$

corresponding to the known 26] relation between scattering angle and impact parameter in the generalized AS metric:

$$
\tan \vartheta / 2=-f^{\prime} / 2
$$

In that saddle approximation we can also use:

$$
\lim _{\nu \rightarrow \infty} \frac{1}{\nu} \int_{0}^{\nu} \mathrm{d} \nu^{\prime} \exp \left[i \nu^{\prime}\left(q_{T}-f^{\prime} E\right)^{2}\right]=1
$$

and therefore obtain for the scattering amplitude

$$
G_{c}\left(p, p^{\prime} / A S\right)=4 E \delta\left(q_{u}\right) \int \mathrm{d}^{2} b \exp \left[-i q_{T} \cdot b+i E f(b)\right],
$$

in agreement with the known result [27]. 


\subsection{Scattering by a black hole}

The problem of the scattering of a massless scalar particle by a (Schwarzschild) black hole is most easily discussed in spherical coordinates. We introduce the Green function for the partial waves $(E, l, m)$ and $\left(E^{\prime}, l^{\prime}, m^{\prime}\right)$ as

$$
G\left(E, l, m, E^{\prime}, l^{\prime}, m^{\prime}\right)=\int \mathrm{d}^{4} x \int \mathrm{d}^{4} y\left\langle l^{\prime} m^{\prime} E^{\prime} \mid y\right\rangle\langle x \mid l m E\rangle G(x, y)
$$

for which, in standard notation,

$$
\begin{aligned}
\langle x \mid l m E\rangle & =Y_{l m}(\phi, \theta) R_{E l}(r) \exp -i E t \\
\left\langle l^{\prime} m^{\prime} E^{\prime} \mid y\right\rangle & =Y_{l^{\prime} m^{\prime}}^{*}(\phi, \theta) R_{E^{\prime} l^{\prime}}^{*}(r) \exp -i E^{\prime} t,
\end{aligned}
$$

in order to transform to spherical coordinates.

In this case the propagator for the partial waves can be written as

$$
\begin{aligned}
G(E & \left., l, m, E^{\prime}, l^{\prime}, m^{\prime}\right)=\lim _{\substack{\nu_{f} \rightarrow+\infty \\
\nu_{i} \rightarrow-\infty}} \frac{1}{\nu_{f}-\nu_{i}} \int \mathrm{d} X^{\mu}\left(\nu_{i}\right) \\
\quad & \times \exp \left[-i E t\left(\nu_{i}\right)+i E^{\prime} t\left(\nu_{f}\right)\right] \\
& \times Y_{l m}\left(\phi\left(\nu_{i}\right), \theta\left(\nu_{i}\right)\right) Y_{l^{\prime} m^{\prime}}^{*}\left(\phi\left(\nu_{f}\right), \theta\left(\nu_{f}\right)\right) R_{E l}\left(r\left(\nu_{i}\right)\right) R_{E^{\prime} l^{\prime}}^{*}\left(r\left(\nu_{f}\right)\right),
\end{aligned}
$$

where the measure is

$$
\mathrm{d} X^{\mu}=\mathrm{d} t\left(\nu_{i}\right) r^{2}\left(\nu_{i}\right) \mathrm{d} r\left(\nu_{i}\right) \mathrm{d} \phi\left(\nu_{i}\right) \mathrm{d} \cos \theta\left(\nu_{i}\right)
$$

and one integrates over the initial conditions, the final ones being given by the dynamics.

The classical equations of motion are given in parametric form as [28]:

$$
\begin{aligned}
\left(\frac{\mathrm{d} r}{\mathrm{~d} \nu}\right)^{2} & =1-\frac{b^{2}}{r^{2}}\left(1-\frac{r_{0}}{r}\right), \\
\frac{\mathrm{d} \theta}{\mathrm{d} \nu} & =\frac{b}{r^{2}} \\
\frac{\mathrm{d} t}{\mathrm{~d} \nu} & =\left(1-\frac{r_{0}}{r}\right)^{-1},
\end{aligned}
$$

where $b=l / E$ and $r_{0}=2 M G$ is the horizon radius.

We consider a planar motion with $m=0$ and

$$
\phi\left(\nu_{i}\right)=\phi\left(\nu_{f}\right),
$$

which implies that the integration over the azimuthal initial angle gives a $\delta_{0, m^{\prime}}$. 
The integration over the angle $\theta\left(\nu_{i}\right)$ can also be performed directly, to yield

$$
\delta_{l l^{\prime}} i^{2 l} P_{l}(\cos \Delta \theta)
$$

where

$$
\Delta \theta=\theta\left(\nu_{i}\right)-\theta\left(\nu_{f}\right)
$$

Similarly, we can integrate over the initial times to obtain

$$
\int \mathrm{d} t\left(\nu_{i}\right) \exp \left[-i\left(E-E^{\prime}\right) t\left(\nu_{i}\right)+i E^{\prime} \Delta t\right]=\delta\left(E-E^{\prime}\right) \exp [i E \Delta t]
$$

where

$$
\Delta t=t\left(\nu_{f}\right)-t\left(\nu_{i}\right)
$$

In this way we find:

$$
\begin{aligned}
& G(l, E)=\lim _{\substack{\nu_{f} \rightarrow+\infty \\
\nu_{i} \rightarrow-\infty}} \frac{1}{\nu_{f}-\nu_{i}} \int r^{2}\left(\nu_{i}\right) \mathrm{d} r\left(\nu_{i}\right) i^{2 l} \exp [i E \Delta t] \\
& \quad \times P_{l}(\cos \Delta \theta) R_{E l}\left(r\left(\nu_{i}\right)\right) R_{E l}^{*}\left(r\left(\nu_{f}\right)\right),
\end{aligned}
$$

which, for large $l$, can be approximated by

$$
\begin{aligned}
& G(l, E)=\lim _{\substack{\nu_{f} \rightarrow+\infty \\
\nu_{i} \rightarrow-\infty}} \frac{i^{2 l}}{\nu_{f}-\nu_{i}} \int \frac{r\left(\nu_{i}\right) \mathrm{d} r\left(\nu_{i}\right)}{r\left(\nu_{f}\right)} \\
& \quad \times \exp i\left[E \Delta t-p_{r} r\left(\nu_{f}\right)-p_{r}^{\prime} r\left(\nu_{i}\right)-l \pi\right] \\
& \quad \times\left[\exp \left(i l \Delta \theta+i \frac{\pi}{4}\right)-\exp \left(-i l \Delta \theta-i \frac{\pi}{4}\right)\right],
\end{aligned}
$$

where we have taken, respectively, an incoming wave at the initial time and an outgoing wave at the final time. In our parametrization, and for $\nu_{i}$ and $\nu_{f}$ sufficiently large, we have $r\left(\nu_{i}\right) \simeq r\left(\nu_{f}\right)=L$, in such a way that the argument of the exponential no longer depends on $r\left(\nu_{i}\right)$. Therefore, the remaining integration over $r\left(\nu_{i}\right)$ can be performed to give

$$
\frac{1}{\nu_{f}-\nu_{i}} \int \mathrm{d} r\left(\nu_{i}\right) \simeq \frac{1}{\nu_{f}-\nu_{i}} \int_{\nu_{i}}^{\nu_{f}} \mathrm{~d} L \longrightarrow 1,
$$

and $2 L \simeq \nu_{f}-\nu_{i}$. The radial momenta are

$$
p_{r} \simeq p_{r}^{\prime}=E \sqrt{L^{2}-b^{2}} / L \simeq E L .
$$


The partial wave amplitude (propagator) $S(l, E)$ is thus given by:

$$
S(l, E)=G(l, E)=\exp \left(2 i \delta_{l}\right)
$$

with:

$$
2 \delta_{l}=\frac{E}{\hbar}[\Delta t-2 L] \pm\left[l(\Delta \theta+\pi)+\frac{\pi}{4}\right] .
$$

Equation (4.34) is the main result of this section. It expresses the phase shifts in terms of $\Delta t$ and $\Delta \theta$, which are defined in (4.28) and (4.26) and can be obtained by integrating the classical equations of motion (4.23).

The free-motion phase shift is just

$$
2 \delta_{l}^{(0)}=\pi l+\frac{\pi}{4} \text {. }
$$

Going back to momentum space, the propagator can now be written as

$$
G(E, \vartheta / S c h w)=\sum_{l}(2 l+1) P_{l}(\cos \vartheta) G(l, E)
$$

where $\vartheta$ is the scattering angle.

The sum over $l$ is dominated by a stationary phase at

$$
\pm \vartheta=\frac{\mathrm{d} \delta_{l}}{\mathrm{~d} l}
$$

By using the explicit form of $\delta_{l}$, (4.34), we find the stationary phase condition (4.37) in the form $(L \rightarrow \infty)$ :

$$
\pm \vartheta=b \int_{r_{\min }}^{\infty} \frac{\mathrm{d} r}{r^{2} \sqrt{1-\left(1-r_{0} / r\right)\left(b^{2} / r^{2}\right)}}-\pi
$$

which agrees with the classical equation for the orbit found in [28] in terms of an elliptic integral of the first kind.

The shift in the time coordinate in (4.34) is particularly interesting. It can be written as

$$
\Delta t=\nu_{f}-\nu_{i}+2 r_{0} \int_{r_{\min }} \frac{\mathrm{d} r}{r-r_{0}} \frac{1}{\sqrt{1-\left(1-r_{0} / r\right)\left(b^{2} / r^{2}\right)}} .
$$

We see that for $r_{\text {min }} \leq r_{0}$ (corresponding to angular momenta $l<E \tilde{b}$, where $\tilde{b}=3 \sqrt{3} r_{0} / 2$ is the critical impact parameter below which classical capture takes place), $\Delta t$ acquires an imaginary part:

$$
\operatorname{Im} \Delta t=2 \pi r_{0} .
$$


Thus the incoming wave is almost completely absorbed for $b<\tilde{b}$ (recall that, in order for our approximations to be valid, we are always at $r_{0} E>>1$ ), the transmission probability being $\mathcal{O}\left(e^{-4 \pi r_{0} E}\right)=e^{-E / T_{H}}$, where

$$
T_{H}=1 / 4 \pi r_{0}
$$

is the black hole temperature.

Finally, the imaginary part (4.40) gives an inelastic contribution to partial wave cross sections with $l<E \tilde{b}$ :

$$
\sigma_{i n}^{l}=\frac{\pi}{E^{2}}(2 l+1)\left[1-\exp \left(-E / T_{H}\right)\right] .
$$

The above results are in full agreement with those obtained in the literature by a more direct (and involved) computation [29]. In particular, the total inelastic cross section can easily be evaluated to give

$$
\sigma_{i n}=\frac{\pi}{E^{2}} \sum_{l=0}^{l=E \tilde{b}}(2 l+1) \simeq \frac{27}{4} \pi r_{0}^{2}
$$

In closing this section we wish to mention that sometime ago Hartle and Hawking [30] addressed the problem of spontaneous black hole radiation by using an approach where complex trajectories dominate the path integral.

\section{Two-Body Collisions}

After having checked our method in the case of scattering in an external field, we shall now turn to our real goal, the Planckian-energy collision of two light (massless) quanta.

As explained elsewhere [1, 7], the semiclassical approximation should be valid in the Planckian energy regime for any finite value of the parameter $R / b$ and provides an $\mathcal{S}$-matrix of the form:

$$
\ln \mathcal{S}(E, b)=\frac{i G s}{\hbar} F(R / b, \log s, \log b)(1+\mathcal{O}(\hbar)) .
$$

This approximation corresponds to resumming all Feynman graphs which become (possibly disconnected) tree diagrams once the scalar-particle propagators are cut. They are illustrated in figs. 1 and 2 for the case of elastic and inelastic scattering, respectively. Notice that neither matter loops nor pure graviton loops are 
allowed in these diagrams. Simple power counting arguments show [1, 7] that these loops only contribute to the $\mathcal{O}(\hbar)$ corrections indicated in (5.1).

In the functional integral, the semiclassical limit corresponds to a saddle point approximation in which the classical solution for $X^{\mu}$ and $g_{\mu \nu}$ are inserted in the action. The basic idea is thus quite simple: use the homogeneity in $g_{\mu \nu}$ of the bulk terms in the gravitational and scalar particle actions to reduce the whole action on the classical equations of motion to just surface terms (the gravitational term $S_{K}$ and the term $\left.S_{p_{e x t}}\right)$.

We first discuss the case of elastic scattering, reproducing in a simple and elegant way the known leading-eikonal result, and then briefly discuss how the method retains its validity beyond such approximation.

\subsection{Elastic Scattering}

In the absence of matter loops, the elastic scattering amplitude is given by

$$
\begin{gathered}
(2 \pi)^{4} \delta^{(4)}\left(p_{1}+p_{2}-p_{1}^{\prime}-p_{2}^{\prime}\right) A\left(p_{1}+p_{2} \rightarrow p_{1}^{\prime}+p_{2}^{\prime}\right)= \\
\quad \times \int\left[\mathrm{d} g_{\mu \nu}\right] \exp \left(i S_{g}\right) G_{c}^{1}\left(p_{1}, p_{1}^{\prime} / g\right) G_{c}^{2}\left(p_{2}, p_{2}^{\prime} / g\right) .
\end{gathered}
$$

where, for the sake of simplicity, we restrict our attention to the case of two distinguishable scalar particles, thus avoiding complications with crossed or annihilation diagrams.

By means of (3.14), the right-hand side of eq. (5.2) can be written as

$$
\lim _{\substack{\nu_{i} \rightarrow \infty \\ p_{i}^{2} \rightarrow 0}}\left(\frac{-i}{\nu_{1}}\right)\left(\frac{-i}{\nu_{2}}\right) \int\left[\sqrt{-g\left(X_{1}\right)} \mathrm{d} X_{1}^{\mu}\right]\left[\sqrt{-g\left(X_{2}\right)} \mathrm{d} X_{2}^{\mu}\right]\left[\mathrm{d} g_{\mu \nu}\right] \exp (i S)
$$

for

$$
S=S_{g}+S_{1}+S_{2}+S_{p_{e x t}}
$$

where

$$
\begin{aligned}
S_{g} & =-\frac{1}{2 \kappa^{2}} \int \sqrt{-g} \mathcal{R} \mathrm{d}^{4} x+S_{K} \\
S_{i} & =\frac{1}{2} \int_{0}^{\nu_{i}} \mathrm{~d} \tau_{i} g_{\mu \nu}\left(X_{i}\right) \dot{X}_{i}^{\mu}\left(\tau_{i}\right) \dot{X}_{i}^{\nu}\left(\tau_{i}\right), \\
S_{p_{\text {ext }}} & =p_{1} \cdot X_{1}(0)-p_{1}^{\prime} \cdot X_{1}\left(\nu_{1}\right)+p_{2} \cdot X_{2}(0)-p_{2}^{\prime} \cdot X_{2}\left(\nu_{2}\right),
\end{aligned}
$$

and $S_{K}$ is the surface term (2.15). 
The classical equations of motion are obtained by a variation with respect to $g_{\mu \nu}:$

$$
\mathcal{R}^{\mu \nu}(x)-\frac{1}{2} g^{\mu \nu} \mathcal{R}(x)=-\frac{\kappa^{2}}{2 \sqrt{-g}} \sum_{i=1,2} \int_{0}^{\nu_{i}} \mathrm{~d} \tau_{i} \delta^{(4)}\left(x-X_{i}\left(\tau_{i}\right)\right) \dot{X}_{i}^{\mu}\left(\tau_{i}\right) \dot{X}_{i}^{\nu}\left(\tau_{i}\right)(5
$$

and with respect to $X_{i}^{\mu}$ :

$$
\ddot{X}_{i}^{\mu}+\Gamma_{\rho \sigma}^{\mu}\left(X_{i}\right) \dot{X}_{i}^{\rho} \dot{X}_{i}^{\sigma}=0 \quad \text { for } \quad i=1,2 .
$$

As we have already discussed in the case of the propagation in an external field, the homogeneity of $S_{i}$ and, now, of $S_{g}$ as well, yields an action that reduces, on the equations of motion, to a surface term:

$$
\left.S\right|_{s o l^{\prime} n}=S_{K}+S_{p_{e x t}}
$$

In the semiclassical limit, we therefore have

$$
\begin{aligned}
& (2 \pi)^{4} \delta^{(4)}\left(p_{1}+p_{2}-p_{1}^{\prime}-p_{2}^{\prime}\right) A\left(p_{1}+p_{2} \rightarrow p_{1}^{\prime}+p_{2}^{\prime}\right)= \\
& \quad \lim _{\substack{\nu_{i} \rightarrow \infty \\
p_{i}^{2} \rightarrow 0}} \frac{-1}{\nu_{1} \nu_{2}} \int \mathrm{d} X_{1}(0) \mathrm{d} X_{1}\left(\nu_{1}\right) \mathrm{d} X_{2}(0) \mathrm{d} X_{2}\left(\nu_{2}\right) \exp \left(\left.i S\right|_{s o l^{\prime} n}\right) .
\end{aligned}
$$

The amplitude (5.9) can be rewritten by introducing the variables

$$
\begin{aligned}
y_{i} & =X_{i}(0)-X_{i}\left(\nu_{i}\right) \\
\Delta & =\left(X_{1}(0)+X_{1}\left(\nu_{1}\right)\right) / 2-\left(X_{2}(0)+X_{2}\left(\nu_{2}\right)\right) / 2 \\
q & =\left(p_{1}-p_{1}^{\prime}\right) / 2-\left(p_{2}-p_{2}^{\prime}\right) / 2 \\
P_{i} & =\left(p_{i}+p_{i}^{\prime}\right) / 2 \\
\Sigma & =\sum_{i} X_{i}
\end{aligned}
$$

after which an integration over $\Sigma$ cancels the overall $\delta$-function on the left-hand side of eq. (5.9). We thus obtain

$$
\begin{aligned}
& A\left(p_{1}+p_{2} \rightarrow p_{1}^{\prime}+p_{2}^{\prime}\right)= \\
& \quad \lim _{\substack{\nu_{i} \rightarrow \infty \\
p_{i}^{2} \rightarrow 0}} \frac{-1}{\nu_{1} \nu_{2}} \int \mathrm{d}^{4} \Delta \exp \left[i q \cdot \Delta+i P_{1} \cdot y_{1}+i P_{2} \cdot y_{2}+i S_{K}\right] .
\end{aligned}
$$

It is at this stage that (possibly non-perturbative) approximate solutions for the classical collision geometries or their generalizations should be used to calculate the amplitude (5.11). In this paper we only carry out a first non-trivial check of the method by showing how the known leading eikonal result arises from our surface terms. The derivation is then compared with the one using perturbation theory in the Newton constant. 


\subsection{Leading Eikonal Approximation}

The leading eikonal approximation corresponds, in our approach, to working out the surface terms appearing in (5.11) to $\mathcal{O}\left(E^{2}\right)$. For the external particle contribution the calculation is simple since, to this order, one just needs the shifts $y_{i}$ to $\mathcal{O}(E)$. To this purpose we can use the leading order form of the metric which consists of the two independent AS shock waves created by the two particles:

$$
\begin{aligned}
h_{\mu \nu}= & h_{\mu \nu}(1)+h_{\mu \nu}(2) \\
= & -\frac{\kappa^{2} E_{1}}{2 \pi} \tilde{\nabla}_{\mu} U \tilde{\nabla}_{\nu} U \delta(U) \ln \left(y^{2}+z^{2}\right) \\
& -\frac{\kappa^{2} E_{2}}{2 \pi} \tilde{\nabla}_{\mu} V \tilde{\nabla}_{\nu} V \delta(V) \ln \left((y-b)^{2}+z^{2}\right),
\end{aligned}
$$

where, in suitable coordinates, the transverse vector $b$ can be identified with the transverse components of the four-vector $\Delta$ appearing in (5.11).

The calculation of the shifts is now identical to the one used in section 4.2 , in the case of scattering from an external field. In a generic boosted frame, in which the two particles are coming against each other along the $x$ axis, the initial momenta are

$$
p_{1}^{\prime} \simeq p_{1}=\left(E_{1}, E_{1}, 0,0\right)
$$

and

$$
p_{2}^{\prime} \simeq p_{2}=\left(E_{2},-E_{2}, 0,0\right)
$$

and we readily find (see eq.(4.4):

$$
P_{1} \cdot y_{1}=P_{2} \cdot y_{2}=-\frac{\kappa^{2} E_{1} E_{2}}{2 \pi} \ln b^{2} .
$$

We see that the contribution of the external momenta is twice the desired value because, unlike in the external metric problem, there are now two identical contributions from each colliding particle. If the known result is to emerge, the gravitational surface term $S_{K}$ should contribute as:

$$
S_{K}=\frac{\kappa^{2} E_{1} E_{2}}{2 \pi} \ln b^{2},
$$

We shall first show how this result arises very simply from the Bondi masses and next compare it with the perturbative calculation. An apparent difficulty in both approaches is that we need the action to $\mathcal{O}\left(E^{2}\right)$ whereas the metric (5.12) is 
valid only to to $\mathcal{O}(E)$. Nevertheless, as we shall see, having the metric to $\mathcal{O}(E)$ is actually sufficient.

We recall, from the appendix, the meaning of the Bondi masses. $M_{-}(v)$, at $\mathcal{I}^{-}$, is the energy brought into the system by the (advanced) time $v$, while $M_{+}(u)$, at $\mathcal{I}^{+}$, is the energy present in the system at (retarded) time $u$. Note the difference in notation and meaning: the arguments $v$ and $u$ of the Bondi masses are exactly null variables and label, respectively, converging and diverging spherical congruences, whereas the $V$ and $U$ coordinates appearing in (5.12) are only approximately null (they are such only in the flat metric) and label plane congruences.

To lowest order in $E_{1}, E_{2}$, the only relevant effect is again the above mentioned shift of the geodesics computed this time in terms of the variables $u, v$. Consider first the case of a single non-interacting massless particle. In this case, $U$ remains exactly null and the Bondi masses are of the form:

$$
M_{-}(v)=E_{1} \theta(v), M_{+}(u)=E_{1} \theta(-u), \quad\left(E_{2}=0\right),
$$

expressing the fact that a particle of energy $E_{1}$ comes in through $\mathcal{I}^{-}$at the instant $v=0$ and goes out through $\mathcal{I}^{+}$at the instant $u=0$. There are no further energy fluxes either through $\mathcal{I}^{-}$or through $\mathcal{I}^{+}$.

In the case of two particles, there are still no interactions at past null infinity. Therefore the Bondi mass $M_{-}$is simply

$$
M_{-}(v)=E_{1} \theta(v)+E_{2} \theta(v),
$$

which means that both particles show up at $\mathcal{I}^{-}$at the instant $v=0$.

The time instants of their arrival at $\mathcal{I}^{+}$will now be shifted in comparison with (5.17). By using the geodesic shifts in $U$ and $V$ and the asymptotic relation between the two sets of coordinates, it is easy to show that the first particle will arrive at $\mathcal{I}^{+}$at $u=u_{1}$ and the second at $u=u_{2}$ where

$$
\begin{aligned}
& u_{1}=-\frac{\kappa^{2} E_{2}}{2 \pi} \ln b^{2}, \\
& u_{2}=-\frac{\kappa^{2} E_{1}}{2 \pi} \ln b^{2} .
\end{aligned}
$$

The Bondi mass at $\mathcal{I}^{+}$is, therefore,

$$
M_{+}(u)=E_{1} \theta\left(u_{1}-u\right)+E_{2} \theta\left(u_{2}-u\right) .
$$


It should be stressed here that, while (5.18) is an exact expression, (5.20) accounts only for the lowest-order effect of the time shift and neglects higher-order contributions from gravitational radiation and mass renormalization.

Inserting eqs.(5.18) and (5.20) into the expression (2.15) for $S_{K}$ :

$$
S_{K}=-\frac{1}{2} \int_{-\infty}^{+\infty} \mathrm{d} x\left[M_{+}(-x)-M_{-}(x)\right]
$$

we obtain precisely the desired result (5.16). Note that the time shifts (5.19) are to be computed from the metric only to $\mathcal{O}(E)$ since there is already a factor $E$ in the Bondi masses. This is the way a metric of $\mathcal{O}(E)$ leads to an action of $\mathcal{O}\left(E^{2}\right)$. With Bondi masses limited to first order in $E$, one would have for $M_{+}$an expression similar to (5.18) and a vanishing result for $S_{K}$, in complete agreement with the fact that there is no contribution linear in $E$ in the action.

The perturbative calculation proceeds instead as follows. The surface term $S_{K}$ is transformed back to the volume integral of a total derivative (see (2.12) after using Gauss' theorem) yielding

$$
S_{K}=\frac{1}{2 \kappa^{2}} \int_{\Omega} \mathrm{d}^{4} x \sqrt{-\tilde{g}}\left(\tilde{\nabla}^{2} \tilde{g}^{\mu \nu} h_{\mu \nu}-\tilde{\nabla}^{\mu} \tilde{\nabla}^{\nu} h_{\mu \nu}\right)
$$

where the tilde stands for flat space-time.

For a direct calculation of (5.22) we would need a solution to second order in $E$ since, again, the insertion of the lowest-order solution (5.12) in (5.22) yields zero. This problem can be avoided, however, by noticing that for a traceless source - such as our two massless particles-

$$
\mathcal{R}=\left(\tilde{\nabla}^{2} \tilde{g}^{\mu \nu}-\tilde{\nabla}^{\mu} \tilde{\nabla}^{\nu}\right) h_{\mu \nu}+\mathcal{O}\left(h_{\mu \nu}^{2}\right)=0
$$

and therefore $S_{K}$ can be written as the quadratic part of the Hilbert action plus higher-order terms, that is

$$
\begin{aligned}
S_{K}= & \frac{1}{8 \kappa^{2}} \int_{\Omega} \mathrm{d}^{4} x \sqrt{-\tilde{g}} h_{\mu \nu}\left[\tilde{g}^{\mu \alpha} \tilde{g}^{\nu \beta} \tilde{\nabla}^{2}+\tilde{g}^{\alpha \beta} \tilde{\nabla}^{\mu} \tilde{\nabla}^{\nu}\right. \\
& \left.-2 \tilde{g}^{\mu \alpha} \tilde{\nabla}^{\nu} \tilde{\nabla}^{\beta}-\tilde{g}^{\mu \nu} \tilde{g}^{\alpha \beta} \tilde{\nabla}^{2}+\tilde{g}^{\mu \nu} \tilde{\nabla}^{\alpha} \tilde{\nabla}^{\beta}\right] h_{\alpha \beta}+\mathcal{O}\left(h_{\mu \nu}^{3}\right) .
\end{aligned}
$$

The metric (5.12) is now sufficient because (5.24) is quadratic in the solution. We thus obtain that

$$
S_{K}=\frac{1}{8 \kappa^{2}} \int \mathrm{d}^{4} x \sqrt{-\tilde{g}} h_{\mu \nu}\left(\tilde{g}^{\mu \alpha} \tilde{g}^{\nu \beta} \tilde{\nabla}^{2}\right) h_{\alpha \beta}=\frac{\kappa^{2} E_{1} E_{2}}{2 \pi} \ln b^{2},
$$


since in this case

$$
\tilde{g}^{\alpha \beta} h_{\alpha \beta}=\tilde{\nabla}^{\alpha} h_{\alpha \beta}=0
$$

We have thus obtained, by two conceptually different calculations, the same final result for the surface action $S_{K}$. Comparison of the two methods confirms the validity of our approach. It also shows, in our opinion, that working in terms of the Bondi masses is simpler and physically more meaningful. The crucial test will come, of course, with non-perturbative computations, something we do not want to attempt here.

The scattering amplitude (5.11) can now be computed. In order for the two particles to scatter at all, we have to impose the two conditions

$$
X_{2}^{-}\left(\nu_{2}\right)>X_{1}^{-}(0), \quad X_{1}^{+}\left(\nu_{1}\right)>X_{2}^{+}(0) \text {. }
$$

This gives two $\theta$-functions that can be written in terms of the variable $\Delta$ as

$$
\theta\left(\Delta^{+}-E_{1} \nu_{1}\right) \theta\left(\Delta^{-}-E_{2} \nu_{2}\right) \text {. }
$$

The condition (5.28) enforces the requirement that between $\nu=0$ and $\nu=\nu_{1}$, as well as between 0 and $\nu_{2}$, the two trajectories cross each other. The integration over the light-like components of $\Delta$ thus gives

$$
\begin{gathered}
\int \mathrm{d} \Delta^{+} \mathrm{d} \Delta^{-} \exp \left[\frac{i}{2} q_{+} \Delta^{+}+\frac{i}{2} q_{-} \Delta^{-}\right] \theta\left(\Delta^{+}-E_{1} \nu_{1}\right) \theta\left(\Delta^{-}-E_{2} \nu_{2}\right)= \\
4\left(\frac{\exp \left(\frac{i}{2} q_{+} E_{1} \nu_{1}\right)-1}{i q_{+}}\right)\left(\frac{\exp \left(\frac{i}{2} q_{-} E_{2} \nu_{2}\right)-1}{i q_{-}}\right) \stackrel{q_{+}, q_{-} \rightarrow 0}{\longrightarrow} E_{1} E_{2} \nu_{1} \nu_{2}
\end{gathered}
$$

canceling the factor $1 / \nu_{1} \nu_{2}$ in (5.9). We therefore obtain:

$$
A\left(p_{1}+p_{2} \rightarrow p_{1}^{\prime}+p_{2}^{\prime}\right)=E_{1} E_{2} \int \mathrm{d}^{2} b \exp \left[i q_{T} \cdot b-i \frac{\kappa^{2} E_{1} E_{2}}{2 \pi} \ln b^{2}\right],
$$

in agreement with previous results [1, 3, 4, 5, 6].

\subsection{Inclusion of Gravitational Bremsstrahlung}

In order to extend the calculations of the previous section to higher orders in $R / b$, one has to deal with the problem of gravitational bremsstrahlung (see fig. 2). Equivalently, in the language of particle physics, one encounters infrared 
divergences in amplitudes with a given number of final gravitons and some BlockNordsiek-type treatment becomes necessary in order to be able to extract finite, physically meaningful results [7].

The general reasoning given in section 2.2 insures that the surface nature of the semiclassical $\mathcal{S}$-matrix remains valid even after allowing for the effects of gravitational radiation. It is however instructive to see how this works in detail in the case of two-body inelastic collisions within a conventional LSZ treatment of the $\mathcal{S}$-matrix.

According to LSZ the amplitude for two scalar particles $(\psi)$ to scatter with emission of $n$ gravitons $(h)$ is given (symbolically) by

$$
A(2 \rightarrow 2+n)=\left.\langle\psi \psi \psi \psi h h \cdots h\rangle\right|_{\mathrm{LSZ}}
$$

that is by applying the standard LSZ steps (Fourier transform, truncation of propagators and going on shell) to the $4+n$-point function shown in (5.31).

These amplitudes are generated by the functional

$$
A(2 \rightarrow 2, J)=\left.\langle\psi \psi \psi \psi \exp (h J)\rangle\right|_{\mathrm{LSZ}}
$$

through standard functional differentiation with respect to the external source $J$. In the absence of scalar-field loops, the LSZ operation amounts to replacing pairs of scalar fields by the corresponding amputated propagators $G_{c}$ and the source $J$ by $\Delta^{(2)} h_{a s}(x)$, where $\Delta^{(2)}$ is the quadratic operator appearing in eq. (5.25). $h_{a s}(x)$ then becomes the new external source.

Finally, in order for the produced gravitons to be on-shell, the condition

$$
\Delta^{(2)} h_{a s}(x)=0
$$

has to be imposed at the end of the computation. This allows us to replace $h(x)$ in eq. (5.32) with the full $g_{\mu \nu}(x)$.

We conclude that a power expansion in $h_{a s}$ of the $\mathcal{S}$-matrix functional

$$
\mathcal{W}\left(h_{a s}\right)=\int\left[\mathrm{d} g_{\mu \nu}\right] \exp \left[i S_{g}+i S_{b r}\right] G_{c}^{1}\left(p_{1}, p_{1}^{\prime} / g\right) G_{c}^{2}\left(p_{2}, p_{2}^{\prime} / g\right),
$$

where

$$
S_{b r}=\int \mathrm{d}^{4} x g(x) \Delta^{(2)} h_{a s}(x),
$$

gives the amplitudes for emission of any number of gravitons. Following the procedure of Section 5.1, we can rewrite (5.34) as a functional integral over $g_{\mu \nu}, X_{1}^{\mu}$ and 
$X_{2}^{\nu}$ with a modified action $S$ containing, beside the terms in (5.4), the additional bremsstrahlung term (5.35).

The crucial observation at this point is that the additional term (5.35) is still effectively homogeneous of degree one in $g_{\mu \nu}$. Therefore, within the stated approximations, we are still left with the result (5.8) and

$$
\mathcal{W}\left(h_{a s}\right)=\exp \left[\left.i\left(S_{K}+S_{p_{e x t}}\right)\right|_{s o l^{\prime} n}\right],
$$

as expected from the general arguments of section (2.2)

Note that, while the two surface terms occurring in (5.36) are still the same ones as in the elastic case, their actual values now include, implicitly, a dependence on gravitational radiation through $h_{a s}(x)$. Giving $h_{a s}(x)$ amounts to specifying a definite final coherent state of gravitons, the closest one can get quantum-mechanically to a classical radiation field. For a generic $h_{a s}(x)$, one will still be plagued by infrared problems. However, as in the analogue QED problem, a well-defined coherent state for which infrared divergences cancel will be chosen by the dynamics and will represent quantum mechanically the classical asymptotic gravitational radiation field generated in the collision.

In concluding this section, we want again to remark that the validity of (5.8) does not depend on any approximation except the tree approximation, for both the gravitational field and the first-quantized particles. The surface term has one and the same form for any interactions of the gravitating fields but its value is different for different interactions.

To what extent the surface nature of gravity persist when graviton loops are taken into account is discussed in the next section.

\section{Inclusion of graviton loops}

The extension of the above ideas beyond the classical approximation for the metric is based on [32, 33, 34] which dealt with the quantum fluctuations of the conformal mode of the gravitational field. In this section we first give a new, more direct, derivation of the result of these works that also shows at which points care should be taken when using these results; next, we discuss its relevance for the present work.

Let us write

$$
g_{\mu \nu}(x)=\bar{g}_{\mu \nu}(x) \phi^{2}(x),
$$


singling out the conformal mode of the gravitational field, and impose on $\bar{g}_{\mu \nu}(x)$ a constraint

$$
\chi(\bar{g}(x))=0,
$$

such that the equation

$$
\chi\left(\Omega^{2}(x) \bar{g}(x)\right)=0,
$$

with $\bar{g}$ satisfying (6.2), has only one solution

$$
\Omega^{2}(x)=1 \text {. }
$$

We may then reparametrize the ten components of $g_{\mu \nu}$ into nine independent components of $\bar{g}_{\mu \nu}$, constrained by condition (6.2), and the conformal mode $\phi(x)$.

With respect to $\bar{g}_{\mu \nu}$, eq. (6.2) plays the role of a gauge-fixing condition, which removes the arbitrariness of local conformal transformations $\bar{g}_{\mu \nu} \rightarrow \Omega^{2} \bar{g}_{\mu \nu}$. We shall consider only the transformations that become the identity at the asymptotically flat infinity; the boundary conditions for the conformal mode are

$$
\left.\phi(x)\right|_{\mathcal{I}^{-}, \mathcal{I}^{+}, I^{\circ}}=1 .
$$

The choice of the gauge-fixing function $\chi(\bar{g})$ is limited only by the requirement that the continuous matrix

$$
\bar{Q}(x, y)=\bar{g}_{\mu \nu}(x) \frac{\delta \chi(\bar{g}(y))}{\delta \bar{g}_{\mu \nu}(x)}
$$

be invertible.

Our goal is to rewrite the functional integral (2.16) in terms of a set of variables containing the conformal mode $\phi(x)$ explicitly. However, instead of introducing $\phi$ and the nine independent components of $\bar{g}_{\mu \nu}$, we introduce eleven variables - $\phi$ and the ten components of $\bar{g}_{\mu \nu}$-and insert the delta-function $\delta(\chi(\bar{g}))$ in the measure. By calculating the relevant Jacobians, we find

$$
\prod_{x, \mu \leq \nu} \mathrm{d} g_{\mu \nu}=(\operatorname{det} \bar{Q}) \prod_{x} \phi^{19} \delta(\chi(\bar{g})) \mathrm{d} \phi \prod_{\mu \leq \nu} \mathrm{d} \bar{g}_{\mu \nu}
$$

with $\bar{Q}$ given by (6.6). The measure in (2.16) contains local factors in $g_{\mu \nu}$ [31] which, after the substitution of (6.1), will change the total power of $\phi$ in the transformed measure. However, in what follows, we shall ignore the local factors in the measure altogether by assuming, for instance, dimensional regularization. 
Next, let us find the form of the action (2.3) in the new variables. We have

$$
\frac{1}{6} \sqrt{-g} \mathcal{R}(g)=\sqrt{-\bar{g}}\left(\phi \bar{\nabla}^{2} \phi+\frac{1}{6} \mathcal{R}(\bar{g}) \phi^{2}\right)
$$

where the quantities and operators with a bar refer to $\bar{g}_{\mu \nu}$. The transformation of the boundary term $S_{K}[g]$ is also non-trivial, and its effect is that the total action contains no second derivatives of $\phi$ :

$$
\begin{aligned}
& -\frac{1}{2 \kappa^{2}} \int \mathrm{d}^{4} x \sqrt{-g} \mathcal{R}+S_{K}[g]= \\
& \quad-\frac{3}{\kappa^{2}} \int \mathrm{d}^{4} x \sqrt{-\bar{g}}\left[-(\bar{\nabla} \phi)^{2}+\frac{1}{6} \mathcal{R}(\bar{g}) \phi^{2}\right]+S_{K}[\bar{g}]
\end{aligned}
$$

so that its variational equation in $\phi$ is of the form

$$
\left(\bar{\nabla}^{2}+\frac{1}{6} \mathcal{R}(\bar{g})\right) \phi=0 .
$$

As a result, the functional integral (2.16) for the case of pure gravity takes the form

$$
\begin{aligned}
\mathcal{S}= & \int\left[\mathrm{d} \bar{g}_{\mu \nu}\right][\mathrm{d} \phi](\operatorname{det} \bar{Q}) \delta(\chi(\bar{g})) \exp \left\{-\frac{3 i}{\kappa^{2}} \int \mathrm{d}^{4} x \sqrt{-\bar{g}}\left[-(\bar{\nabla} \phi)^{2}\right.\right. \\
& \left.\left.+\frac{1}{6} \mathcal{R}(\bar{g}) \phi^{2}\right]+i S_{K}[\bar{g}]\right\} .
\end{aligned}
$$

The conformal mode can now be integrated out. Because of the boundary condition (6.5), one must first make a shift

$$
\phi(x)=\phi_{0}(x \mid \bar{g})+\varphi(x)
$$

where $\phi_{0}(x \mid \bar{g})$ is a solution of eqs. (6.10), (6.5), and

$$
\left.\varphi(x)\right|_{\mathcal{I}^{-}, \mathcal{I}^{+}, I^{\circ}}=0 .
$$

We have

$$
\phi_{0}(x \mid \bar{g})=1+\frac{1}{6} \bar{G} \overline{\mathcal{R}}
$$

where $\bar{G}$ is the Green function

$$
\bar{G}=-\left(\bar{\nabla}^{2}+\frac{1}{6} \overline{\mathcal{R}}\right)^{-1}
$$

whose boundary conditions, apart from the fact that the contraction $\bar{G} \overline{\mathcal{R}}$ vanishes at infinity, are to be discussed below. 
Equation (6.11) takes the form

$$
\begin{aligned}
\mathcal{S}= & \int\left[\mathrm{d} \bar{g}_{\mu \nu}\right][\mathrm{d} \varphi](\operatorname{det} \bar{Q}) \delta(\chi(\bar{g})) \exp \left\{i S_{\text {conf } f}[\bar{g}]-\right. \\
& \left.-\frac{3 i}{\kappa^{2}} \int \mathrm{d}^{4} x \sqrt{-\bar{g}} \varphi\left(\bar{\nabla}^{2}+\frac{1}{6} \mathcal{R}(\bar{g})\right) \varphi\right\}
\end{aligned}
$$

with

$$
S_{\text {conf }}[\bar{g}]=-\frac{1}{2 \kappa^{2}} \int \mathrm{d}^{4} x \sqrt{-\bar{g}}\left[\mathcal{R}(\bar{g})+\frac{1}{6} \mathcal{R}(\bar{g}) \bar{G} \mathcal{R}(\bar{g})\right]+S_{K}[\bar{g}] .
$$

The crucial point is the integration over the field $\varphi$. Its action has a wrong sign, which is a consequence of the fact that, with Euclidean signature of the metric, the Einstein-Hilbert action, even with the term $S_{K}[g]$ added, is unbounded from below [34]. It is the conformal mode that makes it unbounded. The Euclidean functional integral over $\varphi$ diverges exponentially and we must rotate the integration contour to imaginary $\varphi$ [34]. In the Lorentzian context, the prescription to be used is different: one must take for the propagator of the field $\varphi$, instead of the Feynman's Green function its complex conjugate:

$$
\left(\bar{\nabla}^{2}+\frac{1}{6} \overline{\mathcal{R}}\right) \rightarrow\left(\bar{\nabla}^{2}+\frac{1}{6} \overline{\mathcal{R}}-i \epsilon\right) .
$$

One thus obtains

$$
\mathcal{S}=\int\left[\mathrm{d} \bar{g}_{\mu \nu}\right] \delta(\chi(\bar{g}))(\operatorname{det} \bar{Q})\left(\operatorname{det}\left(\bar{\nabla}^{2}+\frac{1}{6} \overline{\mathcal{R}}-i \epsilon\right)\right)^{-1 / 2} \exp \left(i S_{\text {conf }}[\bar{g}]\right) .
$$

The final step is the choice of the gauge-fixing function $\chi(\bar{g})$ satisfying the condition $\operatorname{det} \bar{Q} \neq 0$. Equation (6.19) is valid with any such $\chi(\bar{g})$, but let us choose

$$
\chi(\bar{g})=\mathcal{R}(\bar{g}) .
$$

Then, for $\bar{Q}$ in eq. (6.6), one obtains

$$
\bar{Q}(x, y)=3\left(\bar{\nabla}^{2}-\frac{1}{3} \overline{\mathcal{R}}\right) \delta(x, y) .
$$

Under the condition $\overline{\mathcal{R}}=0$ implied by the delta-function in (6.19), this guarantees that $\operatorname{det} \bar{Q} \neq 0$. Furthermore, under the condition $\overline{\mathcal{R}}=0$, one has from (6.17):

$$
\left.S_{\text {conf }}[\bar{g}]\right|_{\bar{R}=0}=\left.S_{K}[\bar{g}]\right|_{\bar{R}=0},
$$

and the functional integral (6.19) takes the form

$$
\mathcal{S}=\int\left[\mathrm{d} \bar{g}_{\mu \nu}\right] \delta(\mathcal{R}(\bar{g}))\left(\operatorname{det} \bar{\nabla}^{2}\right)^{1 / 2} \exp \left(i S_{K}[\bar{g}]\right)
$$


with only the surface term in the action.

The action $S_{\text {conf } f}[\bar{g}]$ in (6.17) was first obtained in [32] and next discussed in [33, 34]. It is invariant under local conformal transformations that become the identity at the asymptotically flat infinity:

$$
S_{\text {conf }}\left[\Omega^{2} \bar{g}\right]=S_{\text {conf }}[\bar{g}] .
$$

Indeed, the action $S_{\text {conf }}[\bar{g}]$ is obtained from the action of Einstein's theory by making the substitution

$$
\begin{aligned}
& g_{\mu \nu}(x)=\bar{g}_{\mu \nu}(x) \phi_{0}^{2}(x \mid \bar{g}), \\
& S_{\text {conf }}[\bar{g}]=\left.S[g]\right|_{g=\bar{g} \phi_{0}^{2}(\bar{g})}, \\
& S[g]=-\frac{1}{2 \kappa^{2}} \int \mathrm{d}^{4} x \sqrt{-g} \mathcal{R}(g)+S_{K}[g] .
\end{aligned}
$$

Since the function $\phi_{0}(x \mid \bar{g})$ in (6.14) transforms as [32, 33]

$$
\phi_{0}\left(x \mid \Omega^{2} \bar{g}\right)=\Omega^{-1}(x) \phi_{0}(x \mid \bar{g}),
$$

the combination on the right-hand side of (6.25) is conformal-invariant, and hence any functional of this combination is conformal invariant. The action $S_{\text {conf }}[g]$ may be regarded as a conformal-invariant part of Einstein's action. By rewriting eq. (6.17) in the form

$$
S[g]=S_{\text {conf }}[g]+\frac{1}{12 \kappa^{2}} \int \mathrm{d}^{4} x \sqrt{-g} \mathcal{R} G \mathcal{R},
$$

one makes it obvious that Einstein's action is the broken action $S_{\text {conf } f}$ with the gauge-fixing function (6.20) introduced in the action quadratically. Since the action $S_{\text {conf }}[g]$ does not contain the harmful conformal mode, one may expect that, with Euclidean signature of the metric, this action is already positive-definite. This is indeed the case. In fact, by using the conformal invariance, the action $S_{\text {conf } f}[g]$ can be brought to the gauge $\mathcal{R}=0$ where

$$
S_{\text {conf }[}[g]=\left.S_{K}[g]\right|_{R=0},
$$

and it has been proved [35] that

$$
-\left.S_{K}[g]\right|_{R=0} \geq 0, \quad \operatorname{sign} g=(++++) .
$$


In the derivation above, the final result in eq. 6.23 appears to be obtained by identically transforming the original functional integral. However, even apart from the problem with the propagation of the conformal mode, one reserve remains: the bar on the integration variables $\bar{g}_{\mu \nu}$ in eqs. (6.19) and (6.23) is kept because the asymptotic fields for $g_{\mu \nu}$ and $\bar{g}_{\mu \nu}$ need not be one and the same. Equation (6.19) looks like the result of the quantization of the invariant action $S_{\text {conf }}$ obtained by the usual rules of gauge theory with $\delta(\chi)$ the gauge-breaking term and $\operatorname{det} Q$ the ghost term. The only apparent difference from the usual rules is that, because the action $S_{\text {conf }}$ is non-local, there appears an additional invariant measure

$$
\left(\operatorname{det}\left(\nabla^{2}+\frac{1}{6} \mathcal{R}-i \epsilon\right)\right)^{-1 / 2}
$$

However, gravity theory is not conformal-invariant, and the choice of the conformal gauge is relevant for the physical metric. There is no other place in the integral (6.19) except the asymptotic condition where the conformal invariance can be broken. On the other hand, there is one and only one choice of the conformal gauge with which the metric measures distance. For pure gravity or gravity coupled to conformal-invariant matter fields, this choice is $\mathcal{R}=0$. At the classical level, the variational equations of the action $S_{\text {conf }}[g]$, supplemented with the equation $\mathcal{R}=0$, are equivalent to the Einstein equation. It is, therefore, plausible that, for $\bar{g}_{\mu \nu}$ in (6.23), the original asymptotic conditions apply and, generally, $\bar{g}_{\mu \nu}$ in (6.23) can be identified with the operator of the physical metric. The functional integral (6.23), or its generalization to the presence of massless matter, could then be used directly for calculating the expectation values of the gravitational-field observables and the scattering matrix.

The relevance of the result (6.23) is that, after integrating out the conformal mode, the action in the functional integral becomes a pure surface term. Therefore, this action can be written again in terms of Bondi masses, those pertaining, this time, to the virtual gravitational fields that are summed over in the functional integral. By extending the approach advocated in this paper one can try to use the above result to account for the contributions to the scattering amplitude from non-perturbative virtual field configurations. 


\section{Acknowledgments}

We are grateful to D. Amati, M. Ciafaloni and N. Sanchez for interesting discussions. R.P. and G.A.V. acknowledge the CERN Theory Division for the kind hospitality extended to them. G.A.V. also thanks the Physics Department at the University of Naples, where part of this work was done.

R.P. has been partially supported in the framework of the E.C. Research Program "Gauge theories, applied Supersymmetry and Quantum Gravity" with a financial contribution under contract SC1-CT92-0789. G.A.V. has been supported by the Russian Science Foundation under grant 93-02-15594. 


\section{Appendix}

\section{A Calculation of the boundary term}

Let us introduce on each null surface, where $u$ is constant, two coordinates $\phi^{a}, a=1,2$, which label the light rays, and a third coordinate $r$, which is a parameter along the rays. The coordinates $\phi^{a}$ take values on a 2 -sphere and can be chosen so as to ensure orthogonality, that is $\left(\nabla u, \nabla \phi^{a}\right) \equiv 0$. The parameter $r$ can be chosen to be the luminosity distance along the rays by considering the induced metric I' on $u=$ const :

$$
\left.\mathrm{d} s^{2}\right|_{u=c o n s t}=g_{a b} \mathrm{~d} \phi^{a} \mathrm{~d} \phi^{b}, \quad(a, b=1,2),
$$

where

$$
g_{a b}=\left.g_{a b}(\phi, r, u)\right|_{u=c o n s t},
$$

and requiring that the area of the two-dimensional section $u=$ const, $r=$ const be

$$
\int_{2-\text { sphere }} \mathrm{d}^{2} \phi \sqrt{\operatorname{det} g_{a b}}=4 \pi r^{2} .
$$

The functions $u, r, \phi^{a}$ can serve as local coordinates. Four components of the metric in this coordinate frame are already known. Three of them are

$$
\begin{aligned}
g^{u u} & =(\nabla u)^{2} \equiv 0 \\
g^{u \phi^{1}} & =\left(\nabla u, \nabla \phi^{1}\right) \equiv 0, \\
g^{u \phi^{2}} & =\left(\nabla u, \nabla \phi^{2}\right) \equiv 0,
\end{aligned}
$$

and the fourth is fixed by the constraint (A.3). The remaining six components of the metric characterize the gravitational field. We introduce six unknown functions $\Psi, W, \gamma, \delta$ and $U^{a}(a=1,2)$, so that

$$
\begin{aligned}
g^{u r} & =(\nabla u, \nabla r)=\frac{1}{\Psi} \\
g^{r r} & =(\nabla r)^{2}=\frac{1}{\Psi^{2}} W \\
g^{r \phi^{a}} & =\left(\nabla r, \nabla \phi^{a}\right)=\frac{1}{\Psi} U^{a} \quad(a=1,2),
\end{aligned}
$$

\footnotetext{
${ }^{\dagger}$ The induced metric on $u=$ const is two-dimensional because, along the rays, the interval is zero.
} 
where

$$
\phi^{1}=\theta, \phi^{2}=\varphi \quad 0 \leq \theta \leq \pi, \quad 0 \leq \varphi \leq 2 \pi,
$$

and

$$
(\nabla r, \nabla \theta)=\frac{1}{\Psi} U^{\theta}, \quad(\nabla r, \nabla \varphi)=\frac{1}{\Psi} U^{\varphi} .
$$

Next

$$
g^{\phi^{a} \phi^{b}}=\left(\nabla \phi^{a}, \nabla \phi^{b}\right)=g^{a b}=\left(g_{a b}\right)^{-1},
$$

where

$$
\begin{aligned}
g_{a b} \mathrm{~d} \phi^{a} \mathrm{~d} \phi^{b}= & r^{2}\left[\frac{1}{2}\left(e^{2 \gamma}+e^{2 \delta}\right) \mathrm{d} \theta^{2}+\left(e^{\gamma-\delta}-e^{\delta-\gamma}\right) \sin \theta \mathrm{d} \theta \mathrm{d} \varphi\right. \\
& \left.+\frac{1}{2}\left(e^{-2 \gamma}+e^{-2 \delta}\right) \sin ^{2} \theta \mathrm{d} \varphi^{2}\right]
\end{aligned}
$$

and

$$
\operatorname{det} g_{a b}=r^{4} \sin ^{2} \theta
$$

in agreement with (A.3). Then the metric takes the form

$$
\mathrm{d} s^{2}=-W \mathrm{~d} u^{2}+2 \Psi \mathrm{d} u \mathrm{~d} r+g_{a b}\left(\mathrm{~d} \phi^{a}-U^{a} \mathrm{~d} u\right)\left(\mathrm{d} \phi^{b}-U^{b} \mathrm{~d} u\right)
$$

with $g_{a b}$ given by $(\mathrm{A.13})$, and its determinant is

$$
g=\operatorname{det} g_{\mu \nu}=-\Psi^{2} r^{4} \sin ^{2} \theta .
$$

As seen from (A.16), the chart $u, r, \phi^{a}$ breaks down when $\Psi$ becomes zero or infinity. The reason for that can be understood by considering the null geodesic $u=$ const, $\phi^{a}=$ const. Let $\lambda$ be an affine parameter along this geodesic, growing towards the future. From the geodesic equation one finds

$$
\left.\frac{\mathrm{d} r}{\mathrm{~d} \lambda}\right|_{\substack{u=\text { const } \\ \phi=\text { const }}} \propto(\nabla u, \nabla r)=\frac{1}{\Psi}
$$

from which it follows that, at the point where $\Psi$ becomes zero or infinity, $r$ stops being a monotonic parameter along the light ray passing through this point. At this point the light ray hits the apparent horizon. If, during the history of an outgoing light ray, $\mathrm{d} r / \mathrm{d} \lambda$ changes its sign an odd number of times or if $\mathrm{d} r / \mathrm{d} \lambda \rightarrow 0$ as $\lambda \rightarrow \infty$, this light ray will never reach the asymptotic domain of infinite luminosity distance, where the metric becomes flat. By definition, this light ray will not come to $\mathcal{I}^{+}$. 
By considering only a portion of the full congruence of null geodesics that come to $\mathcal{I}^{+}$, we automatically guarantee that the chart $u, r, \phi^{a}$ covers the asymptotic domain near $\mathcal{I}^{+}$. Equation (A.15) gives the general form of an asymptotically flat metric near $\mathcal{I}^{+}$, and $\mathcal{I}^{+}$is reached at the limit $r \rightarrow \infty$ with fixed $u$ and $\phi^{a}$. The remaining arbitrariness in the choice of the variable $u$ is fixed by the normalization condition

$$
\left.(\nabla u, \nabla r)\right|_{\mathcal{I}^{+}}=-1
$$

Then

$$
(\nabla u, \nabla r)=\frac{1}{\Psi}<0
$$

everywhere in the domain covered by the chart $u, r, \phi^{a}$. The $u$ and $\phi^{a}$ act as coordinates on $\mathcal{I}^{+}$, which is topologically a product of the time axis by the 2 sphere. Because of (A.18), the retarded time $u$ grows along $\mathcal{I}^{+}$towards the future and coincides with the proper time of an observer at rest at large and constant $r$. The metric at $\mathcal{I}^{+}$behaves as follows $(r \rightarrow \infty)$

$$
\begin{aligned}
& \left.W\right|_{\mathcal{I}^{+}}=1-\frac{2 G \mathcal{M}_{+}(u, \varphi, \theta)}{r}+\mathcal{O}\left(\frac{1}{r^{2}}\right), \\
& \left.\frac{\gamma+\delta}{2}\right|_{\mathcal{I}^{+}}=\frac{C_{1}^{+}(u, \varphi, \theta)}{r}+\mathcal{O}\left(\frac{1}{r^{2}}\right), \\
& \left.\frac{\gamma-\delta}{2}\right|_{\mathcal{I}^{+}}=\frac{C_{2}^{+}(u, \varphi, \theta)}{r}+\mathcal{O}\left(\frac{1}{r^{2}}\right), \\
& \left.U^{\theta}\right|_{\mathcal{I}^{+}}=\frac{2 \mathcal{N}_{+}^{\theta}(u, \varphi, \theta)}{r^{2}}+\mathcal{O}\left(\frac{1}{r^{3}}\right) \\
& \left.U^{\varphi}\right|_{\mathcal{I}^{+}}=\frac{2 \mathcal{N}_{+}^{\varphi}(u, \varphi, \theta)}{r^{2}}+\mathcal{O}\left(\frac{1}{r^{3}}\right) \\
& \left.\Psi\right|_{\mathcal{I}^{+}}=-1+\mathcal{O}\left(\frac{1}{r^{3}}\right)
\end{aligned}
$$

where $\mathcal{M}_{+}, C_{1}^{+}, C_{2}^{+}, \mathcal{N}_{+}^{\theta}, \mathcal{N}_{+}^{\varphi}$ are finite functions of $u, \varphi, \theta$.

According to (A.8),

$$
\left.(\nabla r)^{2}\right|_{\mathcal{I}^{+}}=1-\frac{2 G \mathcal{M}_{+}(u, \varphi, \theta)}{r}+\mathcal{O}\left(\frac{1}{r^{2}}\right),
$$


and $\mathcal{M}_{+}$averaged over the two-sphere gives

$$
M_{+}(u)=\frac{1}{4 \pi} \int_{0}^{2 \pi} \mathrm{d} \varphi \int_{0}^{\pi} \mathrm{d} \theta \sin \theta \mathcal{M}_{+}(u, \varphi, \theta),
$$

which is the Bondi mass at $\mathcal{I}^{+}$, that is, the energy that remains in the system by the instant $u$ of retarded time. Its limit at $u \rightarrow-\infty$,

$$
M_{+}(-\infty)=M_{0}
$$

is the $A D M$ mass which is the full conserved energy stored in space-time and measured at spatial infinity $I^{\circ}$. The difference

$$
M_{0}-M_{+}(u)=\int_{-\infty}^{u} \mathrm{~d} u\left(-\frac{\mathrm{d} M_{+}}{\mathrm{d} u}\right)
$$

is the energy radiated away through $\mathcal{I}^{+}$by the instant $u$ of retarded time. This is a sum of the energy carried away by the gravitational waves and the energy radiated by massless sources. The energy conservation law following from the Einstein equations in (2.5) is of the form

$$
\begin{aligned}
-\frac{\mathrm{d}}{\mathrm{d} u} M_{+}(u)= & \frac{1}{4 \pi} \int_{0}^{2 \pi} \mathrm{d} \varphi \int_{0}^{\pi} \mathrm{d} \theta \sin \theta\left[\left(\frac{\partial}{\partial u} C_{1}^{+}\right)^{2}+\left(\frac{\partial}{\partial u} C_{2}^{+}\right)^{2}\right] \\
& +\left.\int_{0}^{2 \pi} \mathrm{d} \varphi \int_{0}^{\pi} \mathrm{d} \theta \sin \theta\left[\frac{1}{4} r^{2} \mathcal{T}_{\mu \nu} \nabla^{\mu} v \nabla^{\nu} v\right]\right|_{\mathcal{I}^{+}}
\end{aligned}
$$

where $v=$ const is the null congruence parallel to $\mathcal{I}^{+}$:

$$
(\nabla v)^{2} \equiv 0,\left.\quad(\nabla v, \nabla u)\right|_{\mathcal{I}^{+}}=-2 .
$$

The derivatives $\partial C_{1}^{+} / \partial u$ and $\partial C_{2}^{+} / \partial u$ of the functions appearing in (A.21), (A.22) and (A.30) are the Bondi-Sachs news functions. They determine the manifestly positive energy flux of the gravitational radiation. They are also the data (final data in the case of $\mathcal{I}^{+}$, initial data in the case of $\mathcal{I}^{-}$) for the two degrees of freedom of the gravitational field counted for a three-dimensional point. The three-dimensional points for which the degrees of freedom of the asymptotically flat gravitational field are counted are points of $\mathcal{I}^{+}$or $\mathcal{I}^{-}$. Finally, the last term in (A.30) is the energy flux of the source. It will be non-vanishing at $\mathcal{I}^{+}$only if the source contains a massless component.

The metric near $\mathcal{I}^{-}$is of a form similar to (A.15) but, in this case, one considers a converging null congruence

$$
(\nabla v)^{2} \equiv 0,\left.\quad(\nabla v, \nabla r)\right|_{\mathcal{I}^{-}}=1,
$$


and the limit of infinite luminosity distance $r$ is reached by going along the light rays towards the past. Equations similar to (A.20)-(A.22) and (A.26)-(A.27) define the Bondi mass $M_{-}(v)$ and news functions $\partial C_{1}^{-} / \partial v, \partial C_{2}^{-} / \partial v$ at $\mathcal{I}^{-}$. The Bondi mass $M_{-}(v)$ is the energy brought to the system by the instant $v$ of advanced time, and its limit at $v \rightarrow+\infty$ is the full $A D M$ mass:

$$
M_{-}(+\infty)=M_{0}
$$

The difference $M_{0}-M_{-}(v)$ is the energy brought to the system by the incoming radiation after the instant $v$ of advanced time. Again, this is a sum of the energies of the incoming gravitational radiation and of an incoming flux of massless sources. The energy conservation law at $\mathcal{I}^{-}$is obtained from (A.30) by changing the notation and the sign of the left-hand side.

Of interest are also the limits $M_{-}(-\infty)$ and $M_{+}(+\infty)$. Since we have

$$
\begin{aligned}
& M_{0}=M_{-}(-\infty)+\int_{-\infty}^{\infty} \mathrm{d} v \frac{\mathrm{d} M_{-}}{\mathrm{d} v}, \\
& M_{0}=M_{+}(+\infty)+\int_{-\infty}^{\infty} \mathrm{d} u\left(-\frac{\mathrm{d} M_{+}}{\mathrm{d} u}\right)
\end{aligned}
$$

and the integrals on the right-hand sides are, respectively, the energy brought by radiation and the energy carried away by radiation during the whole history, we conclude that $M_{-}(-\infty)$ is the energy brought to the system by massive (time-like) sources coming from $I^{-}$. Similarly $M_{+}(+\infty)$ is the remainder that goes to $I^{+}$with time-like sources after the total emission of radiation.

By assuming that $\mathcal{T}^{\mu \nu}$ satisfies the dominant energy condition, it has been proved [36] that not only the radiation fluxes, both gravitational and matter, are non-negative, but also that the $A D M$ mass and Bondi masses are always nonnegative, which means, in particular, that the asymptotically flat system cannot radiate more energy than initially stored.

The functions $\mathcal{N}_{+}^{\theta}$ and $\mathcal{N}_{+}^{\varphi}$ appearing in eqs. (A.23) and (A.24) and the functions $\mathcal{N}_{-}^{\theta}$ and $\mathcal{N}_{-}^{\varphi}$ similarly defined at $\mathcal{I}^{-}$, are associated with radiated angular momentum.

To calculate the surface integral (2.12) over the boundary

$$
\mathcal{I}^{-} \cup \mathcal{I}^{+}: \tau(x)=0
$$


one may first go over to the Penrose space where $\mathcal{I}^{-}$and $\mathcal{I}^{+}$really exist as threedimensional null surfaces, but one may also do this calculation directly in the physical space-time by considering the null surfaces close to infinity and, next, going over to the limit of $\mathcal{I}^{-}$or $\mathcal{I}^{+}$respectively. The contribution of $\mathcal{I}^{+}$is then of the form

$$
\left.S_{K}\right|_{\mathcal{I}^{+}}=\lim _{c \rightarrow 0} \frac{1}{16 \pi G} \int \mathrm{d}^{4} x \sqrt{-\tilde{g}} \delta\left(-\frac{1}{v(x)}+c\right) A^{\mu} \tilde{\nabla}_{\mu}\left(-\frac{1}{v(x)}+c\right),
$$

with $v(x)$ defined in eq. (A.31), and

$$
A^{\mu}=\tilde{g}^{\alpha \beta} \tilde{\nabla}^{\mu} h_{\alpha \beta}-\tilde{g}^{\mu \alpha} \tilde{\nabla}^{\beta} h_{\alpha \beta} .
$$

We shall carry out the calculation of (A.37) by using the Bondi-Sachs frame, where the metric has the form (A.15). Accordingly, the flat metric $\tilde{g}_{\mu \nu}$ has the form

$$
\tilde{g}_{\mu \nu} \mathrm{d} x^{\mu} \mathrm{d} x^{\nu}=-\mathrm{d} u^{2}-2 \mathrm{~d} u \mathrm{~d} r+r^{2}\left(\mathrm{~d} \theta^{2}+\sin ^{2} \theta \mathrm{d} \varphi^{2}\right),
$$

and the components of $h_{\alpha \beta}$ that do not vanish identically can be read off from (A.20)-(A.25):

$$
\begin{aligned}
& h_{u u}=\frac{2 G \mathcal{M}_{+}}{r}+\mathcal{O}\left(\frac{1}{r^{2}}\right), \\
& h_{u r}=h_{r u}=\mathcal{O}\left(\frac{1}{r^{3}}\right) \\
& h_{u \theta}=h_{\theta u}=-2 \mathcal{N}_{+}^{\theta}+\mathcal{O}\left(\frac{1}{r}\right) \\
& h_{u \varphi}=h_{\varphi u}=-2 \sin ^{2} \theta \mathcal{N}_{+}^{\varphi}+\mathcal{O}\left(\frac{1}{r}\right), \\
& h_{\theta \theta}=r^{2}\left(\frac{2 C_{1}^{+}}{r}+\mathcal{O}\left(\frac{1}{r^{2}}\right)\right) \\
& h_{\varphi \varphi}=r^{2} \sin ^{2} \theta\left(-\frac{2 C_{1}^{+}}{r}+\mathcal{O}\left(\frac{1}{r^{2}}\right)\right) \\
& h_{\theta \varphi}=h_{\varphi \theta}=r^{2} \sin \theta\left(\frac{2 C_{2}^{+}}{r}+\mathcal{O}\left(\frac{1}{r^{2}}\right)\right) .
\end{aligned}
$$

In the asymptotic domain near $\mathcal{I}^{+}$, eqs. (A.31) are solved by

$$
v(x)=2 r+u, \quad r \rightarrow \infty \text { at fixed } u .
$$

This makes it possible to carry out the integral over $r$ in (A.37) with the aid of the delta-function, and the result is the limit $r \rightarrow \infty$ of the remaining three-dimensional integral:

$$
\left.S_{K}\right|_{\mathcal{I}^{+}}=\lim _{r \rightarrow \infty} \frac{1}{16 \pi G} \int_{0}^{2 \pi} \mathrm{d} \varphi \int_{0}^{\pi} \mathrm{d} \theta \sin \theta \int_{-\infty}^{\infty} \mathrm{d} u r^{2} A^{\mu} \tilde{\nabla}_{\mu}\left(r+\frac{1}{2} u\right) .
$$


For the integrand in (A.48) we have

$$
A^{\mu} \tilde{\nabla}_{\mu}\left(r+\frac{1}{2} u\right)=\left(\frac{1}{2} \frac{\partial}{\partial r}-\frac{\partial}{\partial u}\right)\left(\tilde{g}^{\alpha \beta} h_{\alpha \beta}\right)-\left(\frac{1}{2} \delta_{r}^{\alpha}-\delta_{u}^{\alpha}\right)\left(\tilde{\nabla}^{\beta} h_{\alpha \beta}\right) .
$$

By calculating the covariant derivatives in the metric (A.39), one obtains

$$
\begin{aligned}
\delta_{r}^{\alpha}\left(\tilde{\nabla}^{\beta} h_{\alpha \beta}\right)= & -\partial_{r} h_{u r}-\frac{2}{r} h_{u r}-\frac{1}{r^{3}} h_{\theta \theta}-\frac{1}{r^{3} \sin ^{2} \theta} h_{\varphi \varphi} \\
\delta_{u}^{\alpha}\left(\tilde{\nabla}^{\beta} h_{\alpha \beta}\right)= & -\partial_{r} h_{u u}-\frac{2}{r} h_{u u}+\partial_{r} h_{u r}-\partial_{u} h_{u r}+\frac{2}{r} h_{u r}+\frac{1}{r^{2}} \partial_{\theta} h_{u \theta} \\
& +\frac{1}{r^{2}} \frac{\cos \theta}{\sin \theta} h_{u \theta}+\frac{1}{r^{2} \sin ^{2} \theta} \partial_{\varphi} h_{u \varphi},
\end{aligned}
$$

and

$$
\tilde{g}^{\alpha \beta} h_{\alpha \beta}=-2 h_{u r}+\frac{1}{r^{2}} h_{\theta \theta}+\frac{1}{r^{2} \sin ^{2} \theta} h_{\varphi \varphi} .
$$

It is now easy to insert the asymptotic behaviors (A.40)-(A.46) to get, for (A.49):

$$
\lim _{r \rightarrow \infty} r^{2} A^{\mu} \tilde{\nabla}_{\mu}\left(r+\frac{1}{2} u\right)=-2 G \mathcal{M}_{+}-2 \partial_{\varphi} \mathcal{N}_{+}^{\varphi}-2 \frac{1}{\sin \theta} \partial_{\theta}\left(\sin \theta \mathcal{N}_{+}^{\theta}\right) .
$$

Upon integration over the sphere in (A.48), the contribution of $\mathcal{N}_{+}^{\varphi}, \mathcal{N}_{+}^{\theta}$ vanishes, and we obtain

$$
\left.S_{K}\right|_{\mathcal{I}^{+}}=-\frac{1}{8 \pi} \int_{0}^{2 \pi} \mathrm{d} \varphi \int_{0}^{\pi} \mathrm{d} \theta \sin \theta \int_{-\infty}^{\infty} \mathrm{d} u \mathcal{M}_{+}(u, \varphi, \theta) .
$$

A similar result, but with the opposite sign, is obtained at $\mathcal{I}^{-}$. The final expression for $S_{K}[g]$ can thus be presented in the form

$$
\begin{aligned}
S_{K} & =-\frac{1}{8 \pi}\left(\int_{\mathcal{I}^{+}} \mathcal{M}_{+}-\int_{\mathcal{I}^{-}} \mathcal{M}_{-}\right) \\
& =-\frac{1}{2}\left(\int_{-\infty}^{\infty} \mathrm{d} u M_{+}(u)-\int_{-\infty}^{\infty} \mathrm{d} v M_{-}(v)\right),
\end{aligned}
$$

where $M_{+}(u)$ and $M_{-}(v)$ are the Bondi masses at $\mathcal{I}^{+}$and $\mathcal{I}^{-}$, and the retarded and advanced time are normalized by the conditions (A.18) and (A.32). 


\section{References}

[1] D. Amati, M. Ciafaloni and G. Veneziano, Phys. Lett. 197B (1987) 81; Int. J. Mod. Phys. A3 (1988) 1615.

[2] D. Amati, M. Ciafaloni and G. Veneziano, Phys. Lett. B 216 (1989) 41;

G. Veneziano, in Superstring 89, Texas A. and M. University 1989, eds. R. Arnowitz et al. (World Scientific, Singapore 1990) p. 86.

[3] G.'t Hooft, Phys. Lett. 198B (1987) 61;

see also C. Klimcik, Phys. Lett. B208 (1988) 373.

[4] I. Muzinich and M. Soldate, Phys. Rev. D37 (1988) 353.

[5] E. Verlinde and H. Verlinde, Nucl. Phys. B371 (1992) 246.

[6] A. Bellini et al., Nucl. Phys. B356 (1991) 69.

[7] D. Amati, M. Ciafaloni and G. Veneziano, Nucl. Phys. B347 (1990) 550; Nucl. Phys. B403 (1993) 707.

[8] See, e.g., R.F. Stark and T. Piran, in Proc. of the 14th Yamada Conference on Gravitational Collapse and Relativity, Kyoto 1986, eds. H. Sato and T. Nakamura (WSPC, Singapore 1986) p.249.

[9] P.D. D'Eath, Phys. Rev. D18 (1978) 990

[10] P.D. D'Eath and P.N. Payne, Phys. Rev. D46 (1992) 658, 675 and 694.

[11] D. Amati, M. Ciafaloni and G. Veneziano, Phys. Lett. B289 (1992) 87.

[12] L.N. Lipatov, Nucl. Phys. B365 (1991) 614.

[13] G.W. Gibbons and S.W. Hawking, Phys. Rev. D15 (1977) 2752.

[14] R. Penrose, in Relativity, Groups and Topology, eds. C. DeWitt and B.S. DeWitt (Gordon and Breach, New York 1964)

R. M. Wald, General Relativity (The University of Chicago Press, Chicago 1984)

[15] R. Sachs, in Relativity, Groups and Topology, eds. C. DeWitt and B.S. DeWitt (New York: Gordon and Breach, 1964)

[16] E.S. Fradkin, Proc. Int. Winter School on Theoretical Physics at JINR (Dubna, 1964); Acta Phys. Hung. XIX (1964) 175.

[17] V. Fock, Phys. Zeit. Sowet. Un. 12 (1937) 404;

Y. Nambu, Prog. Theor. Phys. 5 (1050) 82;

J. Schwinger, Phys. Rev. 82 (1951) 64. 
[18] R. P. Feynman, Rev. Mod. Phys. 20 (1948) 327.

[19] G.A. Milekhin and E.S. Fradkin, Soviet Physics JETP 18 (1964) 1323.

[20] See, e.g., B.M. Barbashov, Soviet Physics JETP 21 (1965) 402;

V.N. Pervushin, Theor. Math. Phys. 4 (1970) 643 and references therein.

[21] D. Kabat and M. Ortiz, Nucl. Phys B388 (1992) 570.

[22] H. Cheng and T.T. Wu, Phys. Rev. Lett. 22 (1969) 666;

H. Abarbanel and C. Itzykson, Phys. Rev. Lett. 23 (1969) 53.

[23] P.C. Aichelburg and R.U. Sexl, Gen. Rel. Grav. 2 (1971) 303.

[24] See, for example: L.D. Landau and E.M. Lifshitz, The Classical Theory of Fields (Pergamon Press, Oxford 1975).

[25] R. Iengo and K. Lechner, Nucl. Phys. B335 (1990) 221;

G. Cristofano et al., Phys. Lett. B246 (1990) 45;

M. Fabbrichesi and R. Iengo, Phys. Lett. B264 (1991) 319.

[26] V. Ferrari, P. Pendenza and G. Veneziano, Gen. Rel. Grav. 20 (1988) 1185.

[27] T. Dray and G. 't Hooft, Nucl. Phys. B253 (1985) 173.

[28] C. Darwin, Proc. R. Soc. A249 (1959) 180.

[29] N. Sanchez, Phys. Rev. D18 (1978) 1030 and 1798; see, also, J.A.H. Futterman, F.A. Handler and R.A. Matzner, Scattering from Black Holes (Cambridge University Press, Cambridge 1988).

[30] J.B. Hartle and S.W. Hawking, Phys. Rev. D13 (1976) 2188.

[31] E.S. Fradkin and G.A. Vilkovisky, Phys. Rev. D8 (1973) 4241.

[32] E.S. Fradkin and G.A. Vilkovisky, 8th Intern. Conf. on General Relativity and Gravitation, Abstracts of Contributed Papers (University of Waterloo, Canada 1977) pp. 148-150.

[33] E.S. Fradkin and G.A. Vilkovisky, Phys. Lett. B73 (1978) 209.

[34] G.W. Gibbons, S.W. Hawking and M.J. Perry, Nucl. Phys. B138 (1978) 141.

[35] R.M. Schoen and S.-T. Yau, Phys. Rev. Lett. 42 (1979) 547.

[36] See R.M. Wald, ref. [14], and references therein. 


\section{Figure Captions}

Fig. 1: Tree diagrams (after cutting the scalar propagators) which are resummed by our method in the case of elastic scattering. The blob represents all (including disconnected) trees.

Fig. 2: Same as in fig. 1 for the case of inelastic processes. 